\title{
Counting gamma rays in the directions of galaxy clusters
}

\author{
D. A. Prokhorov ${ }^{1}$ and E. M. Churazov ${ }^{1,2}$ \\ 1 Max Planck Institute for Astrophysics, Karl-Schwarzschild-Strasse 1, 85741 Garching, Germany \\ e-mail: phdmitry@mpa-garching.mpg.de \\ 2 Space Research Institute (IKI), Profsouznaya 84/32, 117997 Moscow, Russia
}

Received 6 August 2013 / Accepted 19 May 2014

\begin{abstract}
Emission from active galactic nuclei (AGNs) and from neutral pion decay are the two most natural mechanisms that could establish a galaxy cluster as a source of gamma rays in the $\mathrm{GeV}$ regime. We revisit this problem by using 52.5 months of Fermi-LAT data above $10 \mathrm{GeV}$ and stacking 55 clusters from the HIFLUCGS sample of the X-ray brightest clusters. The choice of $>10 \mathrm{GeV}$ photons is optimal from the point of view of angular resolution, while the sample selection optimizes the chances of detecting signatures of neutral pion decay, arising from hadronic interactions of relativistic protons with an intracluster medium, which scale with the X-ray flux. In the stacked data we detected a signal for the central 0.25 deg circle at the level of $4.3 \sigma$. Evidence for a spatial extent of the signal is marginal. A subsample of cool-core clusters has a higher count rate of $1.9 \pm 0.3$ per cluster compared to the subsample of non-cool core clusters at $1.3 \pm 0.2$. Several independent arguments suggest that the contribution of AGNs to the observed signal is substantial, if not dominant. No strong support for the large contribution of pion decay was found. In terms of a limit on the relativistic proton energy density, we derived an upper limit of $\simeq 2 \%$ relative to the gas thermal energy density, provided that the spectrum of relativistic protons is hard $\left(s=4.1\right.$ in $\left.\mathrm{d} N \propto p^{-s} \mathrm{~d}^{3} p\right)$. This estimate assumes that relativistic and thermal components are mixed. For softer spectra the upper limits are weaker and equal to $3 \%$ for $s=4.2,4 \%$ for $s=4.3$, and $6 \%$ for $s=4.4$.
\end{abstract}

Key words. galaxies: clusters: general - gamma rays: galaxies: clusters - radiation mechanisms: non-thermal

\section{Introduction}

Galaxy clusters are megaparsec-scale structures that consist of hundreds of galaxies and high-temperature $\left(T_{\text {gas }} \simeq 10^{7}-10^{8} \mathrm{~K}\right)$ sparse highly ionized plasmas filling the space between galaxies (for a review, see Sarazin 1986). X-ray radiation via bremsstrahlung and via ionic emission lines from intracluster plasmas has permitted the study of thermal components of the intracluster medium (ICM). It has been shown that galaxies and plasmas in a galaxy cluster are gravitationally bound to its dark matter halo. The constraints on nonthermal components in galaxy cluster cores can be obtained from a comparison of gravitational potential profiles derived from X-ray and optical data (e.g., see Churazov et al. 2008). Detection of diffuse radio emission from many galaxy clusters provided strong evidence of the presence of relativistic electrons with very high energies (with a Lorentz factor of $\gamma \sim 10^{3}$ ), emitting through the synchrotron mechanism in magnetic fields of the ICM (for a review, see Ferrari et al. 2008). The interaction of relativistic electrons of such energies with cosmic microwave background (CMB) photons may result in hard X-ray emission via the inverse Compton (IC) process, while the IC emission owing to the interaction of more energetic electrons, $\gamma>10^{5}$, with CMB photons may lead to gamma-ray emission (e.g., see Petrosian et al. 2008).

By analogy with the relativistic particle composition in the Milky Way galaxy, one can assume that relativistic protons are also present in galaxy clusters. The collisions of relativistic protons with thermal protons may result in gamma-ray emission via the decay of neutral pions produced in these inelastic protonproton collisions (e.g., see Dermer 1986). The candidates as sources of electrons and protons of very high energies in galaxy clusters are active and normal galaxies, and structure formation shocks (e.g., see Berezinsky et al. 1997). The lifetime of highenergy protons, $E>10 \mathrm{GeV}$, in galaxy clusters exceeds the Hubble time (e.g., see Völk et al. 1996), but the lifetime of very high-energy electrons with $\gamma \sim 10^{5}$, which emit gamma rays via the IC effect, in the ICM is several orders of magnitude shorter than the Hubble time (e.g., see Petrosian et al. 2008). Observations of diffuse radio emission from galaxy clusters hosting radio halos indicate that electrons with $\gamma \sim 10^{3}$ are continuously produced in the ICM and that acceleration mechanisms overtake the electron energy losses (e.g., Brunetti \& Lazarian 2007). Note that gamma rays produced in annihilation of hypothetical dark matter particles, such as WIMPs, can potentially contribute to emission from galaxy clusters (e.g., see Ackermann et al. 2010b).

Clusters of galaxies, which are bright extended X-ray sources, are promising targets for gamma-ray telescopes (e.g., see Michelson et al. 2010), but an observational evidence that gamma rays are emitted by galaxy clusters is still lacking. The Energetic Gamma Ray Experiment Telescope (EGRET) was collecting data on gamma rays (ended in June 2000) ranging from $30 \mathrm{MeV}$ to $30 \mathrm{GeV}$ (for a review, see Thompson 2008) and its successor, the Fermi Large Area Telescope (LAT), is collecting the data covering the energy range from $20 \mathrm{MeV}$ to more than $300 \mathrm{GeV}$ (Atwood et al. 2009) (the science phase of the Fermi mission began on 2008 August 4). The analyses of the EGRET data (Reimer et al. 2003) and of the first 18 months of FermiLAT data (Ackermann et al. 2010a) have allowed us to derive the flux upper limits on gamma-ray emission from individual galaxy clusters. The predicted gamma-ray fluxes from clusters 
are not much lower than those of the observed flux upper limits (Pinzke \& Pfrommer 2010), which suggests that a stacking analysis might be a powerful tool in the search of gamma rays from galaxy clusters (e.g., see Zimmer et al. 2011; Dutson et al. 2013).

At $10 \mathrm{GeV}$, Fermi-LAT provides an increase in the effective area over EGRET by an order of magnitude. Note that the AntiCoincidence Detector, ACD, of EGRET was monolithic, which caused problems above $10 \mathrm{GeV}$ : electromagnetic showers in the calorimeter produced backsplash that made a signal in the ACD, thus tagging high-energy gamma rays incorrectly as charged particles. To avoid this effect, the ACD for Fermi-LAT is divided into many scintillating tiles (see Moiseev et al. 2007) and, therefore, Fermi-LAT provides us with a unique opportunity to study incoming gamma rays with energies above $10 \mathrm{GeV}$. At energies below $\approx 10 \mathrm{GeV}$, the accuracy of the directional reconstruction of photon events detected by Fermi-LAT is limited by multiple scattering, whereas above $\approx 10 \mathrm{GeV}$, multiple scattering is unimportant and the accuracy is limited by the ratio of the silicon-strip pitch to silicon-layer spacing (see Atwood 2007). While our manuscript was in preparation, the FermiLAT catalog of gamma-ray sources at energies above $10 \mathrm{GeV}$ based on the data accumulated during the first three years of the Fermi mission was published (Ackermann et al. 2013). The relatively low intensity of diffuse backgrounds (Galactic, extragalactic isotropic, and residual cosmic rays) at energies above $10 \mathrm{GeV}$ makes source detection possible with only a few gamma rays.

In this paper, we perform a search for gamma-ray emission from a sample of galaxy clusters with modest angular sizes by analyzing the data from Fermi-LAT. For this purpose, we stack photons with $\mathrm{GeV}$ energies coming from the regions covering clusters of galaxies. There are different theoretical spectralspatial models for gamma-ray emission from galaxy clusters, including the gamma-ray production 1) via IC emission of primary electrons or neutral pion decay emission produced in cosmic ray $(\mathrm{CR})$ proton-ICM proton interactions, which are expected to have spectra with photon indices of $\simeq 2$ or steeper (e.g., see Miniati 2003); 2) via very high-energy CR proton-CMB photon pair emission at structure formation shocks with photon indices of $\simeq 1.5$ (e.g., Inoue et al. 2005), and 3) via gamma-ray emission from active galactic nuclei (AGNs) with photon indices of $\simeq 2$ (e.g., Abdo et al. 2009b) ${ }^{1}$. The theoretical argument that gamma-ray photon spectra of galaxy clusters can be harder than those of the Galactic and extragalactic diffuse emission (e.g., see Abdo et al. 2010; Ackermann et al. 2012b) provides us with a lower background at high energies than that at lower energies and, therefore, provides a higher signal-to-noise ratio $(\mathrm{S} / \mathrm{N})$ at high energies. The fine $(0.228 \mathrm{~mm})$ pitch of the strips, applied in Fermi-LAT, gives excellent angular resolution for highenergy photons. The point spread function (PSF) of Fermi-LAT strongly depends on photon energy, and the $68 \%$ angle containment radius significantly decreases with energy (from $\simeq 5^{\circ}$ at $100 \mathrm{MeV}$ to $\simeq 0.2^{\circ}$ at $10 \mathrm{GeV}$, Ackermann et al. (see 2012a)). This allows us to associate the observed photons above $10 \mathrm{GeV}$ more precisely with those that are expected to come from clusters of galaxies, and is especially important for galaxy clusters with an angular size smaller than one degree (i.e., the probability that photons within the PSF come from the source but not from background increases with energy). This association

\footnotetext{
1 Hereafter, neutral pion decay emission and IC emission are attributed to diffuse emission from galaxy clusters, whereas AGN emission is attributed to emission produced in the vicinity of AGNs.
}

permits us to perform a temporal analysis for testing the variability of gamma-ray sources in the directions of galaxy clusters. Below we demonstrate that the number of high-energy photons is sufficient to search for a statistically significant signal from galaxy clusters and that our analysis reveals a tentative excess of gamma rays in the direction of galaxy clusters. Comparing the positions of galaxy clusters in the sky with those of expected gamma-ray blazar sources from the CGRaBS catalog (see Healey et al. 2008), we exclude eight galaxy clusters that are possibly associated with CGRaBS gamma-ray candidate sources and set the upper limit on the ratio of relativistic hadron to thermal energy density using a sample of 47 galaxy clusters.

\section{Observation and data reduction}

The Fermi-LAT, the primary scientific instrument on the Fermi Gamma Ray Space Telescope spacecraft, is an imaging highenergy gamma-ray pair conversion telescope covering the energy range from about $20 \mathrm{MeV}$ to more than $300 \mathrm{GeV}$ (Atwood et al. 2009). Tungsten foils and silicon microstrip detectors are applied to convert incoming photons into electron-positron pairs and to measure the arrival direction of gamma rays. A cesium iodide hodoscopic calorimeter is used to provide an energy measurement of the electron-positron pairs. The Fermi spacecraft travels in an almost circular near-Earth orbit at an altitude of $550 \mathrm{~km}$ with a period of about $96 \mathrm{~min}$. The field of view of Fermi-LAT covers about $20 \%$ of the sky at any time, and it scans continuously, covering the whole sky every three hours (two orbits). By using the silicon strip technique with tungsten convertors (instead of the spark chamber technique), Fermi-LAT achieves a finer precision in the measurement of the directions of incoming gamma rays than that of EGRET. The description of the current instrument performance can be found in (see Ackermann et al. 2012a).

We used the first 52.5 months of the Pass 7 Fermi-LAT data, collected between 2008-08-04 and 2012-11-29, and analyzed the data using the Fermi Science Tools v9r27p1 package ${ }^{2}$."Source" class events, which are recommended for an analysis of gammaray point sources ${ }^{2}$ (including events converted in both the front and back sections of the LAT tracker) with energies between $10 \mathrm{GeV}$ and $270 \mathrm{GeV}$ were selected. Events with zenith angles larger than $100^{\circ}$ were rejected to minimize contamination from gamma rays from the Earth limb. We removed events that occured during satellite maneuvers when the LAT rocking angle was larger than $52^{\circ}$. Time intervals when some event had negatively affected the quality of the LAT data were excluded. We selected events in a circular region of interest of $4^{\circ}$ radius around each galaxy cluster specified below.

The selection of photon events with $E>10 \mathrm{GeV}$ for our counting experiment relied on the higher $\mathrm{S} / \mathrm{N}$ expected in this energy band compared with that at energies of $E>200 \mathrm{MeV}$ and $E>1 \mathrm{GeV}$. The $\mathrm{S} / \mathrm{N}$ for a point-like source observed against an isotropic background is given by

$(S / N)_{\mathrm{E}} \propto N_{\mathrm{src}}(E) \times \sqrt{\frac{A_{\mathrm{eff}}(E)}{I_{\mathrm{bkg}} \times \Sigma}}$,

where $N_{\text {src }}(E)$ is a number of incoming photons with energies $>E$ from the source, $A_{\mathrm{eff}}(E)$ is the effective area of the detector as a function of energy, $I_{\mathrm{bkg}}$ is the intensity of background emission, and $\Sigma$ is the surface area corresponding to the $68 \%$

2 http://fermi.gsfc.nasa.gov/ssc/data/analysis/ 
angle containment radius at energy $E$. Thus one expects the following improvement of the $\mathrm{S} / \mathrm{N}$ using $E>10 \mathrm{GeV}$ instead of $E>200 \mathrm{MeV}$

$$
\frac{(S / N)_{10 \mathrm{GeV}}}{(S / N)_{0.2 \mathrm{GeV}}} \approx\left(\frac{10}{0.2}\right)^{-2.1+1} \sqrt{\frac{0.77}{0.42} \times\left(\frac{10}{0.2}\right)^{(2.4-1)}}\left(\frac{3}{0.25}\right) \approx 3.4,
$$

and using $E>10 \mathrm{GeV}$ instead of $E>1 \mathrm{GeV}$

$$
\frac{(S / N)_{10 \mathrm{GeV}}}{(S / N)_{1 \mathrm{GeV}}} \approx\left(\frac{10}{1}\right)^{-2.1+1} \sqrt{\frac{0.77}{0.68} \times\left(\frac{10}{1}\right)^{(2.4-1)}}\left(\frac{0.9}{0.25}\right) \approx 1.5,
$$

where $0.42,0.68$, and $0.77 \mathrm{~m}^{2}$ are the approximate values of the effective area at $0.2,1$, and $10 \mathrm{GeV}$ for normal incidence photons; $3.0,0.9$, and $0.25^{\circ}$ are the approximate values of the $68 \%$ angle containment radius at $0.2,1$, and $10 \mathrm{GeV}$ (Ackermann et al. 2012a); and 2.1 and 2.4 are power-law photon indices for a point source and isotropic background emission, respectively. To study how the $\mathrm{S} / \mathrm{N}$ changes with energy, we modeled the point source and uniform background spatial maps in four energy bands (1924-3333 MeV, 3333-5773 MeV, $5773 \mathrm{MeV}-10 \mathrm{GeV}$, $>10 \mathrm{GeV}$ ) assuming that both the point source and uniform background are described by power-law spectra. Modeling was performed by using the gtmodel routine from the Fermi Science Tools. The values of photon indices are in the range of [1.5-2.5] for the point source and in the range of [2.3-2.7] for the background. The $68 \%$ angle containment radii at four energies were used to compute the numbers of photons coming from the modeled point source and from the modeled background. The $\mathrm{S} / \mathrm{N}$ for the $E>10 \mathrm{GeV}$ band to that of each of the other three energy bands is shown in Figs. A.1-A.3. These figures show that the $\mathrm{S} / \mathrm{N}$ is highest for the energy band $E>10 \mathrm{GeV}$ for hard photon indices $(\lesssim 2.2)$ of the modeled point source. Note that the different components of background emission, namely extragalactic isotropic background, Galactic foreground, and misidentified charged particles, have different spectral properties. Thus, the use of a single power-law background is an approximation. The spectral analysis of gamma-ray observations of galaxy clusters performed in several energy bands is presented in Sect. 4 to clarify the importance of observed photons with energies of several $\mathrm{GeV}$ for a detection of gamma-ray sources with hard spectra. Performing an analysis in each energy band for each galaxy cluster will allow us to take the spectral properties of the different background components into account.

As mentioned in Sect.1, X-ray astronomy has provided a perfect tool for detecting massive galaxy clusters through imaging and spectroscopy. The ROSAT All-Sky X-ray Survey (RASS; Truemper 1993; Voges et al. 1999) has been conducted for the purpose of searching for X-ray sources. A highly complete flux-limited sample of the X-ray brightest galaxy clusters (HIFLUGCS, the HIghest X-ray FLUx Galaxy Cluster Sample) has been published by Reiprich \& Böhringer (2002). Since the gamma-ray luminosities of galaxy clusters are expected to increase with cluster mass (e.g., see Pinzke \& Pfrommer 2010) and the X-ray luminosities of galaxy clusters increase with cluster mass according to the X-ray luminosity-mass relation (e.g., see Hoekstra et al. 2011), we used the HIFLUGCS catalog to select a sample of galaxy clusters for our analysis of the gammaray data. Note that galaxy clusters from the HIFLUGCS sample are located at high galactic latitudes, $|b|>20^{\circ}$. Therefore, the selection of these clusters for a gamma-ray analysis minimizes contamination of a gamma-ray signal from galaxy clusters by diffuse Galactic emission, which dominates at low galactic latitudes. We present an analysis of gamma-ray emission in the directions of galaxy clusters taking into account the background variation over the sky in Sect. 4.

The HIFLUGCS catalog includes fifty-seven galaxy clusters with angular sizes (defined by the ratio of the virial radius to the distance) smaller than $1^{\circ}$. This catalog also contains several groups and clusters of galaxies with large angular sizes exceeding $1^{\circ}$, namely Fornax, Hydra, Coma, NGC 4636, Centaurus, and NGC 5044. Although these six extended objects are some of the nearest and, therefore, their fluxes are relatively high, we did not include them in the present analysis. This is because their large angular extensions can be problematic to take into account a possible population of extragalactic gamma-ray point sources (e.g., blazars), which can contribute to gamma-ray signals towards these clusters. Possible inhomogeneities of Galactic diffuse emission in the direction of the extended clusters requires a supplementary analysis. The nearby Fornax, Coma, and Centaurus clusters of galaxies are promising targets for gammaray studies conducted with the aim to constrain the parameters of the hadronic cosmic ray model and of some dark matter annihilation models (e.g., Zimmer et al. 2011; Ando \& Nagai 2012). For the remaining sample of fifty-seven galaxy clusters, the mean angular size (i.e., the mean virial radius) is $0.52^{\circ}$ and the standard deviation is $0.15^{\circ}$. Since the standard deviation is significantly smaller than the mean, the same spatial template can be used to fit the counts from these fifty-seven galaxy clusters. These galaxy clusters are the targets of our study. The selection of galaxy clusters with high X-ray fluxes from this catalog provides us with a suitable sample of galaxy clusters to constrain gamma-ray emission that is produced via neutral pion decay, using the proportionality of X-ray and gamma-ray fluxes expected in this model (see, e.g., Ensslin et al. 1997).

The redshifts of these 57 galaxy clusters lie in the interval of $0.016<z<0.2$ (only one cluster lies at $z>0.1$ ) and their X-ray fluxes (in the energy range of $0.1-2.4 \mathrm{keV}$ ) are in the interval of $(2.0-12.1) \times 10^{-11} \mathrm{erg} \mathrm{s}^{-1} \mathrm{~cm}^{-2}$.

A gamma-ray signal from clusters of galaxies can be contaminated by various point gamma-ray sources, for example, blazars, that are occasionally located near galaxy clusters in the sky. To minimize contamination of a signal from galaxy clusters, we explored the LAT two-year Point Source Catalog (the 2FGL catalogue, Nolan et al. 2012) searching for point gamma-ray sources located near the clusters of galaxies from the HIFLUGCS catalog that are detected by the Fermi-LAT in the first two years of the mission. The search showed that there are gamma-ray sources from the 2FGL catalog near the positions of two galaxy clusters (Abell 3376 and Abell 2589). The 2FGL catalog names of these gamma-ray sources are 2FGL $\mathrm{J} 0602.7-4011$ (which lies at a distance of $\approx 0.26^{\circ}$ from the center of Abell 3376) and 2FGL J2325.4+1650 (which lies at a distance of $\approx 0.37^{\circ}$ from the center of Abell 2589). The gamma-ray source 2FGL J2325.4+1650 is associated with the radio source NVSS J232538+164641 in Nolan et al. (2012), while 2FGL J0602.7-4011 is an unidentified gamma-ray source.

To check if these two 2FGL sources can possibly be identified with galaxy clusters, we estimated the flux at $E>200 \mathrm{MeV}$ from each of these 2FGL gamma-ray sources using their fluxes at $E>1 \mathrm{GeV}$ and spectral indices listed in the 2FGL cata$\log$. The calculated fluxes at $E>200 \mathrm{MeV}$ are $6.5 \times 10^{-9}$ and $2.4 \times 10^{-9} \mathrm{ph} \mathrm{cm}^{-2} \mathrm{~s}^{-1}$ for 2FGL J0602.7-4011 and 2FGL $\mathrm{J} 2325.4+1650$, respectively, and are not much lower than the $2 \sigma$ flux upper limit of $4.58 \times 10^{-9} \mathrm{ph} \mathrm{cm}^{-2} \mathrm{~s}^{-1}$ at $E>200 \mathrm{MeV}$ 
for the Coma cluster (taken from Ackermann et al. 2010a). We used the proportionality relation between X-ray and gamma-ray fluxes (expected for gamma-ray emission via pion decay, see e.g., Ensslin et al. 1997) to test the possible identification of these two 2FGL sources with A3376 and A2589. The X-ray fluxes for A3376, A2589, and Coma are taken from Reiprich $\&$ Böhringer (2002) and are equal to $4.6 \times 10^{-11}, 4.7 \times 10^{-11}$, and $6.4 \times 10^{-10} \mathrm{erg} \mathrm{cm}^{-2} \mathrm{~s}^{-1}$, respectively. Taking into account the observed X-ray fluxes and plasma temperatures of A3376, A2589, and Coma, we found that the expected gamma-ray flux from the Coma cluster is about twenty times higher than those expected from A3376 and A2589 when the same ratio of relativistic hadron to thermal energy density is assumed. Since the observed gamma-ray fluxes at $E>200 \mathrm{MeV}$ from these 2FGL sources are not twenty times lower than the flux upper limit for the Coma cluster, we conclude that the upper limit on the ratio of relativistic hadron to thermal energy density obtained from the analysis of the Coma cluster disagrees with the hypotheses that 1) gamma rays from 2FGL J0602.7-4011 and 2FGL $\mathrm{J} 2325.4+1650$ are produced via neutral pion decay emission in A3376 and A2589; and that 2) the ratio of relativistic hadron to thermal energy density in A3376 and A2589 does not exceed the upper limit derived from the analysis of the Coma cluster.

There are $\approx 1000$ BL Lac types of blazars, FSRQ types of blazars, and other AGNs in the 2FGL catalog. Assuming that these extragalactic objects are isotropically distributed in the sky, the expected number of galaxy clusters that have one of these 2FGL gamma-ray point sources within a circle of $0.4^{\circ}$ is $\simeq 0.7$, which agrees with the fact that there are two galaxy clusters from the sample of 57 clusters in the vicinity of one of the 2FGL point gamma-ray sources in the sky. Although the possible association of a gamma-ray source with the cluster Abell 3376 is of interest particularly in light of the ring-shaped nonthermal radio-emitting structures discovered in the outskirts of this cluster (Bagchi et al. 2006) and of the expectation of a strong gamma-ray signal from the outer shock waves of Abell 3376 (e.g. Araudo et al. 2008), we discarded Abell 3376 and Abell 2589 from the sample of the clusters of galaxies selected for the present analysis.

In addition to the point sources located in the close vicinity of galaxy clusters in the sky, there are point sources within the $4^{\circ}$ regions of interest around each cluster. These point sources need to be taken into account to estimate diffuse background emission. The four brightest of these sources included in the 2FGL catalog, 2FGLJ0710.5+5908, 2FGLJ1256.1-0547, 2FGLJ1522.1+3144, and 2FGLJ1800.5+7829, are strong at energies higher than $10 \mathrm{GeV}$. These four sources are associated with blazars, RGB J0710+591, 3C 279, B2 1520+31, and S5 $1803+784$, respectively. The test-statistics values (Mattox et al. 1996) of these four blazars are higher than 100 at $E>10 \mathrm{GeV}$ according to the 2FGL catalog. Since the test-statistics is extensive, the test-statistics are expected to be larger than 200 for each of these four blazars when the first 52.5 months of the Fermi-LAT data are analyzed. Thus, for example, the expected TS value will be equal to 560 for the blazar 2FGLJ1256.1-0547, if we assume that this source is invariable in time. Dividing the expected TS value by the number of the selected regions of interest (i.e., 55), this blazar is expected to appear in the stacked image as a source with a confidence level of $\simeq 3 \sigma$. Note that most of the gamma-ray sources with high galactic latitudes are blazars and that blazars are variable gamma-ray sources in time (e.g. Nolan et al. 2012). Flaring activity of blazars can lead to higher TS values (compared with those listed in the 2FGL catalog) when the 52.5 months of the data are analyzed and,

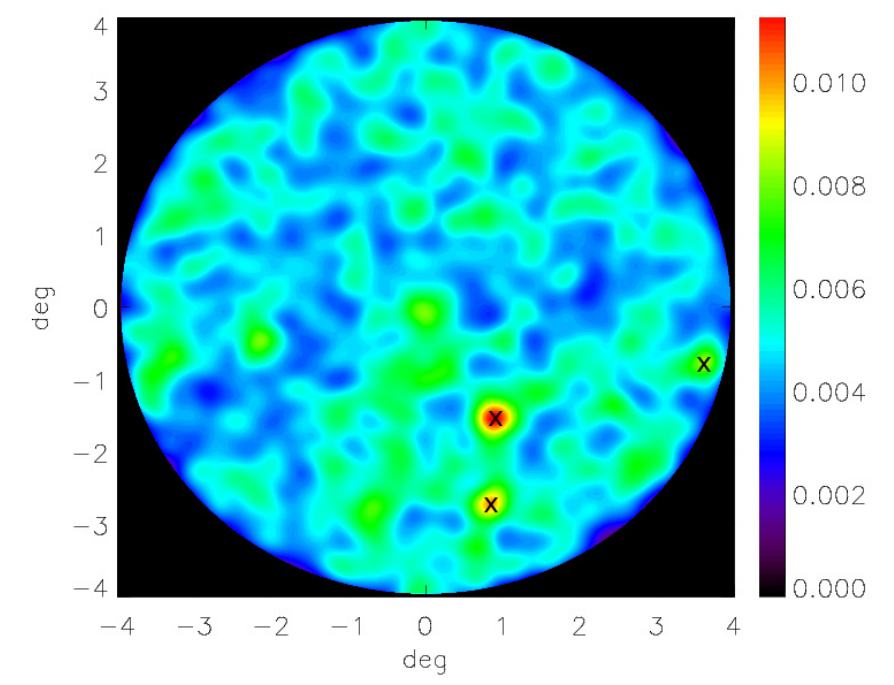

Fig. 1. Gaussian $\left(\sigma=0.15^{\circ}\right)$ kernel smoothed count map (before the subtraction of 2FGL sources) centered on the positions of galaxy clusters for the energy range $10 \mathrm{GeV}-270 \mathrm{GeV}$ and for a pixel size of $0.005^{\circ} \times 0.005^{\circ}$.

therefore, we masked the 2FGL gamma-ray sources to estimate the background emission around the selected galaxy clusters. The masked 2FGL gamma-ray sources are listed in Table A.1. In this table, we show the names of the 2FGL sources, the square root of the TS values at $E>10 \mathrm{GeV}$ (from the 2FGL catalog), and the names of galaxy clusters located within a circle of $4^{\circ}$ radius around each of the sources. To mask these 2FGL sources, we used a circle with a radius of $0.5^{\circ}$, corresponding to the $\approx 90 \%$ angle containment radius, centered on the positions of these sources. The stacked count map of the selected galaxy clusters obtained by combining the count maps for each of the galaxy clusters from our sample with equal weights and smoothed with a Gaussian kernel $\left(\sigma=0.15^{\circ}\right)$ is shown in Fig. 1 (and Fig. 2) before (and after) the subtraction of 2FGL point sources. Each count map is aligned in Galactic coordinates. We did not scale the count maps because the virial radii of the selected galaxy clusters are in a narrow range of $0.4^{\circ}-0.65^{\circ}$. The crosses in Fig. 1 show the positions of bright 2FGL gamma-ray sources, 2FGLJ0710.5+5908, 2FGLJ1256.10547, and 2FGLJ1800.5+7829. Note that the counts of isotropic background emission that were subtracted along with the 2FGL point sources do not strongly affect the estimation of isotropic background emission. This is because the total surface area covered by the masked regions is only $\approx 1.8 \%$ of the total surface area covered by the regions of interest around the selected clusters of galaxies.

Figure 2 demonstrates the excess of the number density of counts towards galaxy clusters (i.e., towards the central region of this count map) over the average number density of counts. Studies of spatial, temporal, and spectral properties of the observed tentative signal towards galaxy clusters are presented below. There are three possible interpretations of this central excess: 1) it is due to gamma-ray emission from galaxy clusters; 2) it is due to a high local concentration of non-2FGL point sources towards galaxy clusters; and 3) it is due to processes occurring in the ICM, but not directly related to galaxy cluster emission. The evaluation of statistical significance of this gamma-ray signal towards galaxy clusters and the study of the origin of the central count density excess is present in the Sect. 3. 


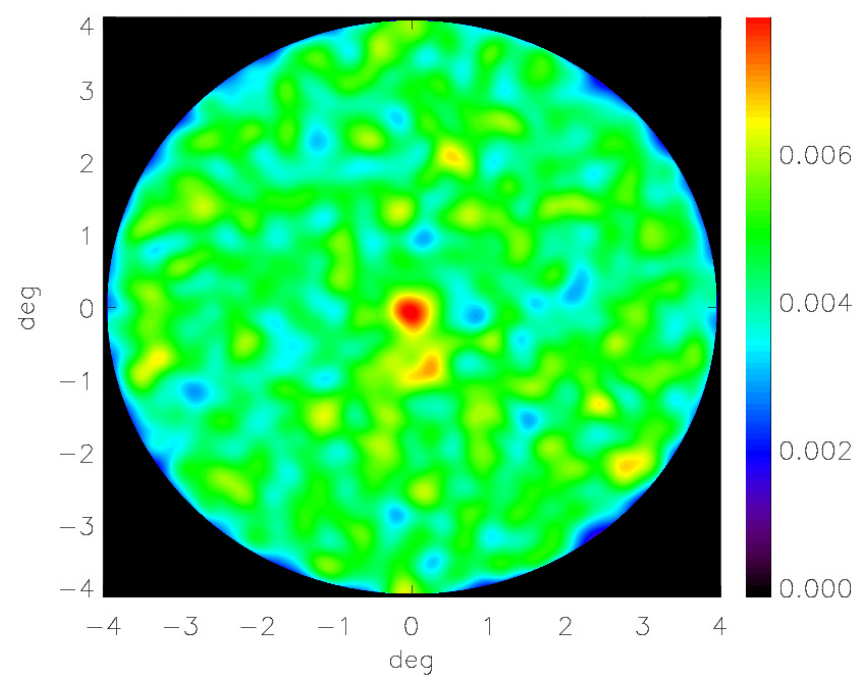

Fig. 2. Gaussian $\left(\sigma=0.15^{\circ}\right)$ kernel smoothed count map (after the subtraction of 2FGL sources) centered on the positions of galaxy clusters for the energy range $10 \mathrm{GeV}-270 \mathrm{GeV}$ and for a pixel size of $0.005^{\circ} \times 0.005^{\circ}$.

We first searched for a signal in excess of the background emission by stacking all galaxy clusters with the same weight. We then used a weighting procedure to place constraints on the neutral pion decay production model. The weighting procedure should lead to a higher $\mathrm{S} / \mathrm{N}$ if the model is correct.

\section{Results}

In this section, we evaluate the statistical significance of the observed gamma-ray signal towards the selected clusters of galaxies and study various interpretations of this signal.

\subsection{Evaluation of the significance of the signal}

To evaluate the statistical significance of the observed gammaray signal towards the selected galaxy clusters, we binned the count map (its smoothed version is shown in Fig. 2) into concentric annuli. We chose the surface area of each of the annuli to be equal to $\pi\left(0.25^{\circ}\right)^{2}$. This value of surface area corresponds the $68 \%$ containment angle at $10 \mathrm{GeV}$. Thus, the first annulus, which covers the center of the count map, should contain $\simeq 68 \%$ of gamma rays incoming from these galaxy clusters if the signal is strongly centrally concentrated. The radius of the k-th annulus is given by $R_{\mathrm{k}}=\sqrt{k} \times 0.25^{\circ}$. Note that the widths of annuli strongly decrease with radius, but this does not affect the estimate of isotropic backround emission. The numbers of photon events in the first twenty annuli (starting from the center of the count map) are shown in Fig. 3. These twenty annuli cover the circular region with a radius of $\approx 1.1^{\circ}$. The radial distribution of photons, shown in Fig. 3, confirms the high count density in the center of the count map. The number of counts in the first annulus equals 63 and is significantly higher than the numbers of counts in other radial bins.

The expected number of photons from background emission needs to be estimated to calculate the significance level of the observed central gamma-ray excess. Since the selected galaxy clusters are at high galactic latitudes $\left(|b|>20^{\circ}\right)$, the background emission can thus be considered as isotropic. We calculated the expected number of background photons in the first annulus. First, we estimated the expected number of background

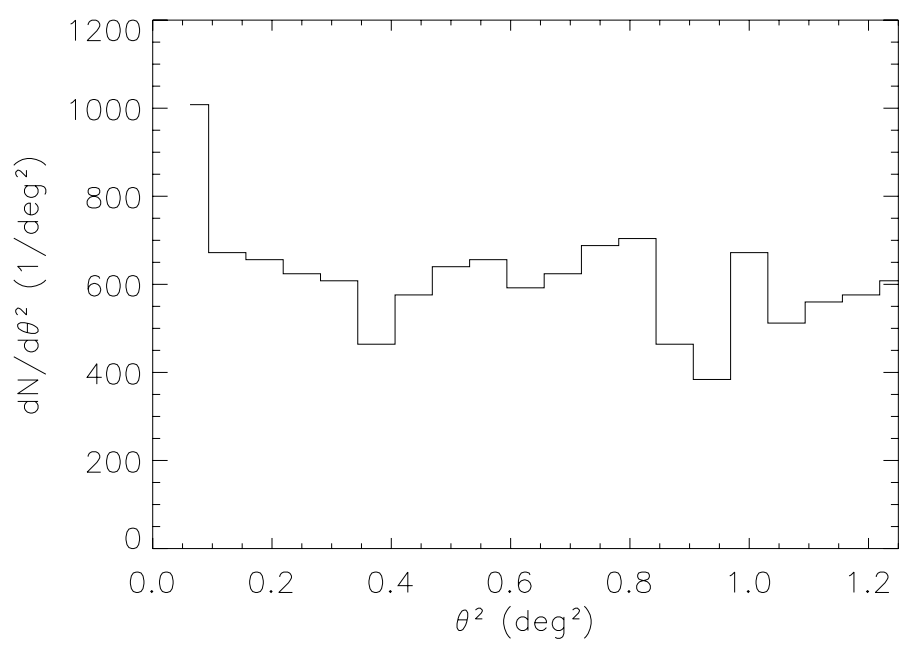

Fig. 3. Radial distribution of photons in annuli with a surface area of $\pi\left(0.25^{\circ}\right)^{2}$.

photons using the nineteen outer annuli. The mean value of photons within an annulus is 37.1 and the standard deviation equals 5.2. Second, we calculated the expected number of background photons taking the regions of $4^{\circ}$ radius and excluding their most central parts of $0.25^{\circ}$. By means of the second method, we found that the mean value of background photons within an annulus is 36.7 and agrees well with that calculated by using the first nineteen annuli. Therefore, the observed number of counts within the first annulus, equal to 63, is significantly higher than the expected number of background counts, i.e., 36.7. The statistical significance level of the count density excess in the first annulus over the expected value equals $(63-36.7) / \sqrt{36.7} \approx 4.3 \sigma$.

We performed the spatial analysis of the central gamma-ray excess to check if the radial distribution of counts agrees with that of a point source or of an extended source. Note that the mean virial radius of the selected galaxy clusters corresponds to $\approx 0.5^{\circ}$ and, therefore, if the observed excess is produced by emission via neutral pion decay, this signal should be modestly extended and its maximal brightness should be at the center. Assuming that the gamma-ray brightness profiles follow those for X-ray brightness derived from a beta model and taking the values of the parameters for a beta model for the selected galaxy clusters from the HIFLUGCS catalog (Reiprich \& Böhringer 2002), we found that the $68 \%$ gamma-ray emission from these clusters should be confined within the central region with a radius of $0.25^{\circ}$. The possible IC gamma-ray emission from the structure formation shocks around galaxy clusters should have an extended profile with maximal brightness at shock fronts. However, if gamma-ray emission from galaxy clusters comes from AGNs located in the most central regions of galaxy clusters (such as NGC 1275 in center of the Perseus cluster Abdo et al. 2009a), the observed radial distribution of counts should be described as a point source. To study the spatial extension of the observed signal towards galaxy clusters, we used three models (a point source for emission from central AGNs, a disk model for emission produced via neutral pion decay, and a thick ring model for emission produced via the IC radiation from structure formation shocks). To perform the spatial analysis, we binned the count map into concentric annuli with a surface area of $\pi \times\left(0.25^{\circ}\right)^{2} / 2$. The numbers of observed counts in the first sixteen annuli from the center are $33,30,30,13,19,21,20,22,20$, $16,16,11,19,16,20$, and 18 . The outer radius of the sixteenth bin is $1^{\circ}$ and corresponds to $\simeq 2 R_{\mathrm{vir}}$. 

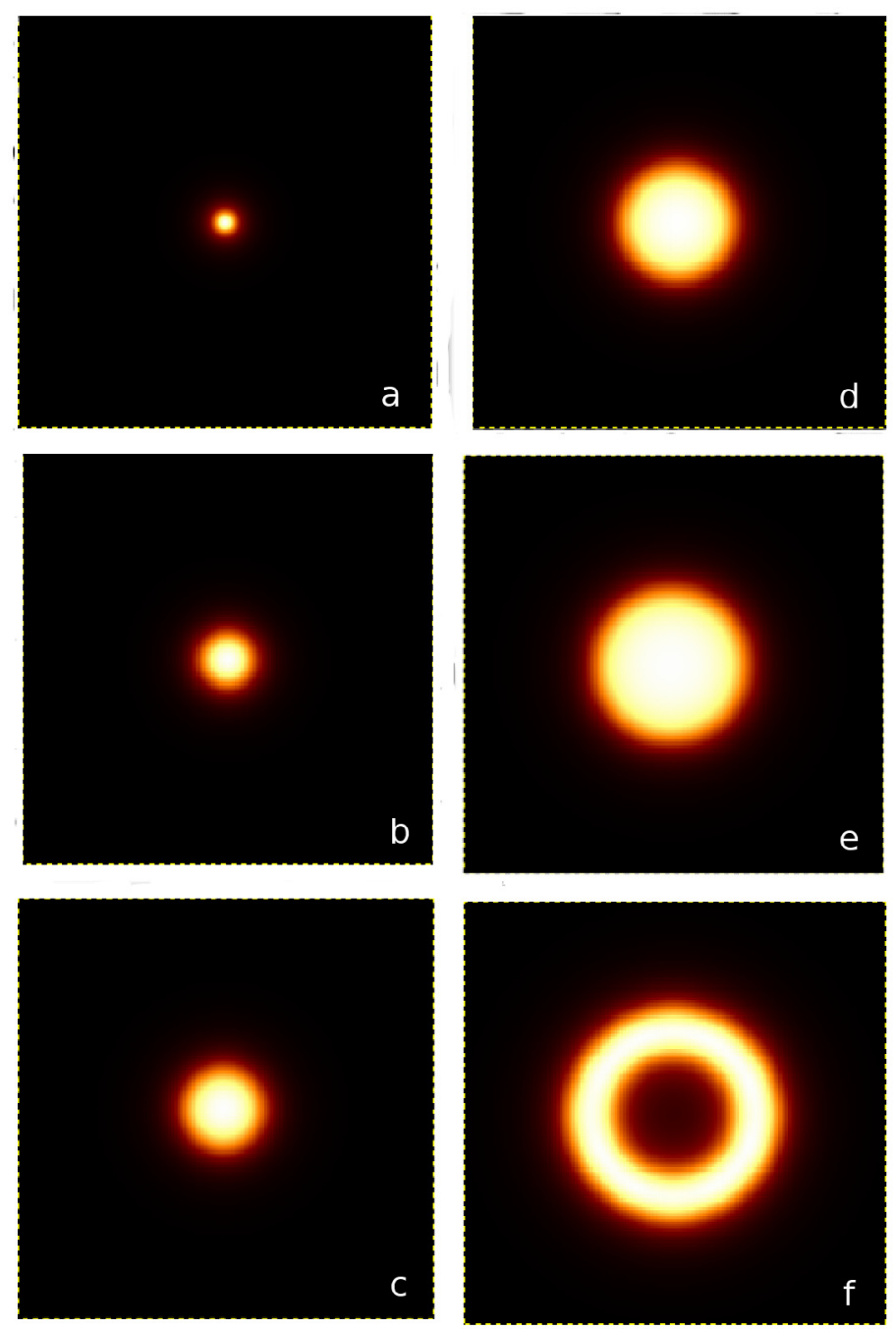

Fig. 4. Two-dimensional templates (with a size of $3 \times 3 \mathrm{deg}^{2}$ ) for the gamma-ray emission from galaxy clusters: a) a point source template; b) -e) templates for emission from a disk of radius $0.15^{\circ}, 0.25^{\circ}, 0.375^{\circ}$, and $\left.0.5^{\circ} ; \mathbf{f}\right)$ a ring-like spatial template.

We produced six spatial templates to estimate the significance level of the observed gamma-ray excess. The first template map describes a point-like source and is obtained by convolving a point source model with the PSF. The following four template maps describe the count distributions for extended disk sources with radii of $0.15^{\circ}, 0.25^{\circ}, 0.375^{\circ}$, and $0.5^{\circ}$ and correspond to the possible neutral pion decay emission from galaxy clusters. The sixth template map describes an extended ring-like profile with inner and outer radii of $0.45^{\circ}$ and $0.65^{\circ}$ (corresponding to $\approx 0.9 R_{\text {vir }}$ and $\approx 1.3 R_{\text {vir }}$, respectively, and corresponds to the possible IC emission from the structure formation shocks around galaxy clusters. All the disk-like and ring-like spatial templates are convolved with the PSF in each energy band. This is needed for the spectral analysis in Sect. 4 to avoid spurious variations of fluxes due to the energy dependence of the PSF. To calculate the PSF, we adopted the power-law spectral distribution for each template with a photon index of -2.1 . The produced templates are shown in Fig. 4. To calculate the number of counts, $N_{\text {src }}$, observed from the gamma-ray source with one of the spatial profiles, shown in Fig. 4, we used the equation

$N_{\mathrm{src}}=\frac{\sum_{k=1}^{16} N_{k} p_{k}}{\sum_{k=1}^{16} p_{k}^{2}}$,

A93, page 6 of 15

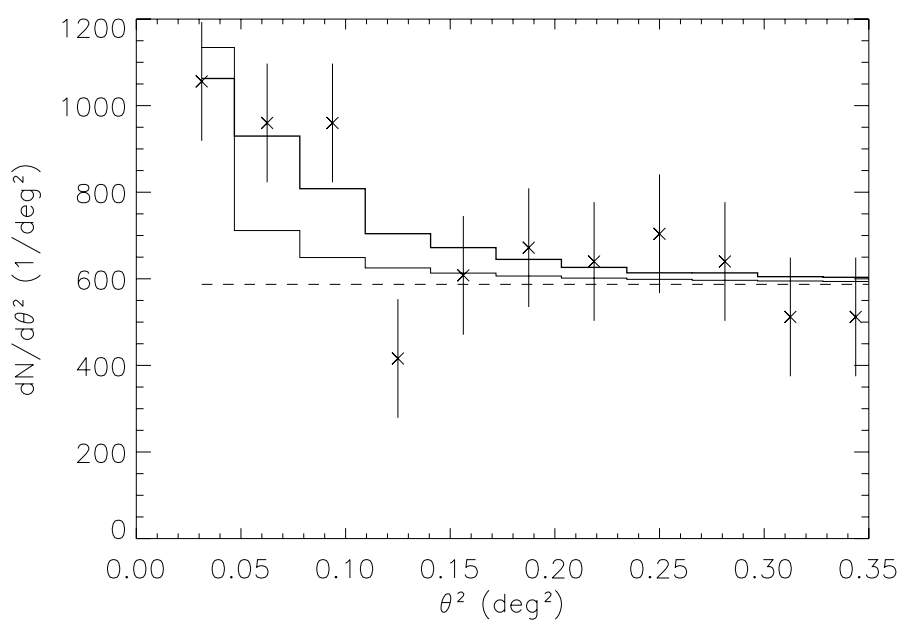

Fig. 5. Best fits of the point source and disk $\left(R=0.25^{\circ}\right)$ source models to the data shown by thin and thick solid lines. The surface area of the annular bin is $\pi\left(0.25^{\circ}\right)^{2} / 2$.

Table 1. Significance of signal detection.

\begin{tabular}{lcc}
\hline \hline Template & Convolution with the PSF & Significance \\
\hline disk $\left(R=0.25^{\circ}\right)$ & no & $4.3 \sigma$ \\
point source & yes & $4.1 \sigma$ \\
disk $\left(R=0.15^{\circ}\right)$ & yes & $4.6 \sigma$ \\
$\operatorname{disk}\left(R=0.25^{\circ}\right)$ & yes & $4.7 \sigma$ \\
$\operatorname{disk}\left(R=0.375^{\circ}\right)$ & yes & $4.2 \sigma$ \\
$\operatorname{disk}\left(R=0.5^{\circ}\right)$ & yes & $3.5 \sigma$ \\
ring $\left(0.45^{\circ}<R<0.65^{\circ}\right)$ & yes & $<1 \sigma$ \\
\hline
\end{tabular}

where $p_{i}$ are weights for each of the annuli determined by the spatial profile for a source model, and $N_{k}$ is the number of observed counts in the $k$ th annulus minus the number of counts from the isotropic background. The best fits of the point source and disk source (with a radius of $0.25^{\circ}$ ) models to the data are shown in Fig. 5 by thin and thick solid lines. The expected number of counts from an isotropic background is shown in Fig. 5 by a dashed line. The number of observed counts in each annular bin along with the errorbars is also plotted in this figure. Using the number of counts observed from the source for each of the models, we calculated the significance level of the observed gamma-ray excess. The calculated value of the significance level is $4.1 \sigma$ for the point source template. The values of the significance level are $4.6 \sigma, 4.7 \sigma, 4.2 \sigma$, and $3.5 \sigma$ for the templates describing gamma-ray emission from disks with radii of $0.15^{\circ}, 0.25^{\circ}, 0.375^{\circ}$, and $0.5^{\circ}$. As for the ring-like model, we found no evidence for IC emission around galaxy clusters and that the observed numbers of counts in outer annuli agree with that expected from background emission within $1 \sigma$. Thus, the model for gamma-ray emission from structure formation shocks cannot account for the observed gamma-ray excess towards the selected galaxy clusters. We list the significance levels for our spectral-spatial templates in Table 1.

We used the sample of 55 galaxy clusters and binned the observed counts in annuli as described above to calculate the Poisson log-likelihood for each of the spectral-spatial models listed in Table 1. Fitting a disk model to the data improves the $\log$-likelihood by $\Delta L \approx 2.5$ compared with the point-source hypothesis. This provides evidence of the spatial extension of the gamma-ray source at a significance level of $\sqrt{2 \times \Delta L} \approx 2.2 \sigma$. 
Table 2. Expected and observed numbers of galaxy clusters with a given number of counts.

\begin{tabular}{lrr}
\hline \hline Numbers of counts & $\begin{array}{r}\text { Expected number } \\
\text { of clusters }\end{array}$ & $\begin{array}{r}\text { Observed number } \\
\text { of clusters }\end{array}$ \\
\hline 0 & 10.5 & 10 \\
1 & 17.4 & 19 \\
2 & 14.4 & 15 \\
3 & 7.9 & 4 \\
4 & 3.3 & 5 \\
5 & 1.1 & 2 \\
6 & 0.3 & 0 \\
\hline
\end{tabular}

Notes. The expected numbers are obtained from the Poisson distribution of counts with parameter $\lambda=1.65$ for all 55 galaxy clusters.

Note that this significance level is not sufficient to claim that the gamma-ray source in the directions of galaxy clusters is extended.

We conclude that the significance level of the central gamma-ray excess is greater than $4 \sigma$ for several spatial models, such as a point-like source model and a disk-like source model.

\subsection{Individual contributions of galaxy clusters to the observed gamma-ray signal}

In this section, we estimate the contribution of each galaxy cluster to the total observed gamma-ray signal towards the selected galaxy clusters and check the consistency of the observed distribution of the number of photon events in galaxy clusters with a Poisson distribution. We also test the homogeneity of incoming photons in time to check if the observed gamma-ray signal towards galaxy clusters can be explained by time-invariant mechanisms of gamma-ray production, such as those via neutral pion decay or via the IC effect.

\subsubsection{Distribution of the number of photon events over the sample of galaxy clusters}

To study the origin of the observed signal towards the selected clusters of galaxies, we analyzed the distribution of counts in galaxy clusters. Note that the first three annular bins, shown in Fig. 5, provide us with the highest $\mathrm{S} / \mathrm{N}$ of $(93-36.7 \times$ $1.5) / \sqrt{36.7 \times 1.5} \approx 5.1$. Thus, for this study we selected counts in the directions of the clusters from our sample within a circle of $0.3^{\circ}$ radius. Below, we calculate the expected number of galaxy clusters with a given number of counts within a circle of $0.3^{\circ}$ radius and compare the expected and observed numbers of galaxy clusters.

The total number of counts in the directions of the galaxy clusters within a circle of $0.3^{\circ}$ radius equals 91 . Therefore, the average number of counts incoming from the direction of a cluster is given by $91 / 55 \approx 1.65$. Assuming a Poisson distribution, we calculated the probability of detecting a given number of counts towards a galaxy cluster. As expected, most of the circular regions $(\simeq 75 \%)$ contain 0,1 , and 2 counts. The expected numbers of clusters with $0-6$ counts within a circular region of $0.3^{\circ}$ radius are shown in Table 2 . If the observed gammaray excess towards the selected galaxy clusters is due to one or two strong gamma-ray sources, the number of circular regions with a high number of counts should be significantly higher than that obtained from an estimate based on the Poisson distribution.
We calculated the observed numbers of clusters with $0-6$ counts within a circular region of $0.3^{\circ}$ and list the observed numbers of counts in Table 2 . We find that there is no circular region with a radius of $0.3^{\circ}$ that contains more than five counts. The difference between the observed number of circular regions with the number of counts, $>2$, and the expected number of such regions obtained from the Poisson distribution with parameter $\lambda=1.65$ is $\approx-2$. We conclude that the observed number of circular regions with a number of counts higher than 2 towards the galaxy clusters is consistent with that obtained from the Poisson distribution with parameter $\lambda=1.65$. Therefore, the contribution of one or two possible strong gamma-ray sources to the observed gamma-ray excess cannot explain the observed excess.

We also calculated the number of circular regions with a given number of counts that are expected from the background emission. The average number of counts within a circular region of $0.3^{\circ}$ radius coming from background emission is $52.8 / 55 \approx$ 0.96. The difference between the observed number of circular regions with the number of counts, $>2$, and the number of such regions expected from background diffuse emission equals $\approx 29$ counts (i.e., $\simeq 55 \%$ of the photons expected from the background emission). We recalculated the significance of detection of a gamma-ray signal towards the selected galaxy clusters excluding the eleven galaxy clusters with the highest observed number of counts within a central circular region of $0.3^{\circ}$ radius and found that the detection significance of the excess decreases from $\simeq 4.7 \sigma$ to $1.5 \sigma$. Therefore, the analysis of these eleven galaxy clusters is important to understand the origin of the observed gamma-ray excess. In Fig. 6 we plot the observed and expected distributions of the numbers of galaxy clusters with a given number of counts. Although the distribution shown in Fig. 6 is well described by a Poisson process, it can also be fitted with several Poisson processes (for an example, it can arise from two samples consisting of forty four galaxy clusters with a Poisson mean of $\lambda_{1} \sim 1.5$ and eleven galaxy clusters with a Poisson mean of $\lambda_{2} \sim 3$ ).

Note that the probability of observing a given number of counts by chance varies from one galaxy cluster to another galaxy cluster and depends on the expected number of counts from the background around each cluster of galaxy. We calculated the expected number of background counts for each of the eleven galaxy clusters with the highest observed number of counts. To calculate the number of background counts for each circular region with a radius of $4^{\circ}$, we masked 2FGL sources. The calculated numbers of background counts are shown in Table 3 for these eleven galaxy clusters. Using the Poisson statistics, we calculated the probability of obtaining the observed number of counts by chance. The obtained probabilities are listed in the fourth column in Table 3. Note that the probability of observing 4 or 5 counts in a circular region of $0.3^{\circ}$ radius by chance is lower than $3 \%$.

\subsubsection{Test for homogeneity of incoming photons in time}

Gamma-ray emission from galaxy clusters produced via neutral pion decay and via the IC emission does not vary with time within the time-range of an observation. We used this argument to check if the observed gamma rays with $E>10 \mathrm{GeV}$ towards the selected galaxy clusters are homogeneously distributed in time. To perform the test, we chose the galaxy clusters with a high number of observed counts in their directions listed in Table 3. In Table A.2 we list the arrival time, given in mission elapsed time (MET) units, and the energy for 


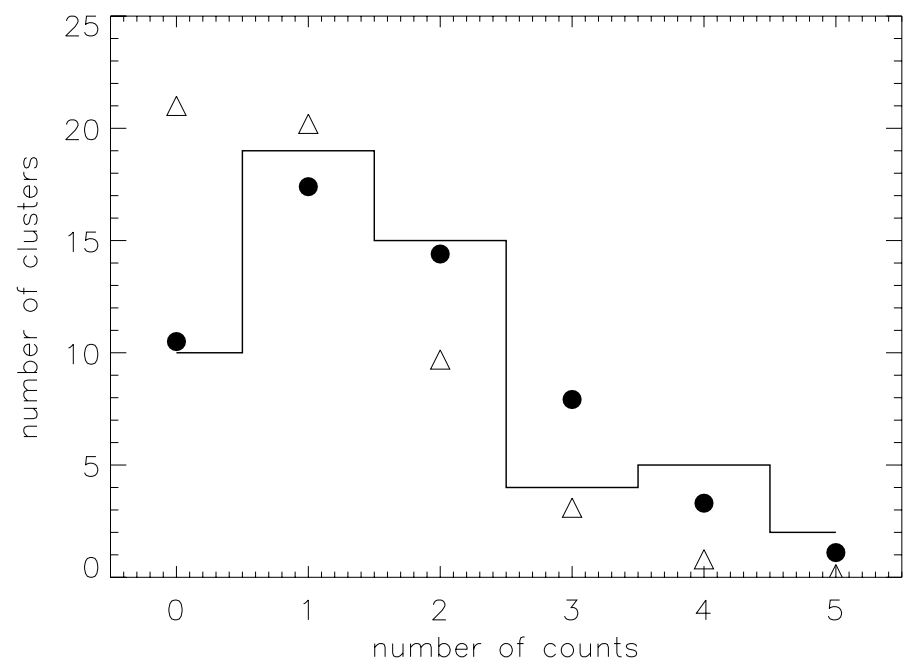

Fig. 6. Observed number of galaxy clusters with a given number of counts (histogram) and the expected numbers from the Poisson distribution with $\lambda=1.65$ (filled circles) and with $\lambda=0.95$ (open triangles).

Table 3. Probability of obtaining the observed number of counts from background emission by chance.

\begin{tabular}{lccr}
\hline \hline Cluster name & Observed counts & Background & Probability, $\%$ \\
\hline A0119 & 3 & 0.73 & 3.1 \\
A3112 & 3 & 0.72 & 3.0 \\
A4038 & 3 & 0.73 & 3.1 \\
ZwIII54 & 3 & 1.33 & 10.4 \\
A0400 & 4 & 1.05 & 1.8 \\
2A0335 & 4 & 1.20 & 2.6 \\
A1367 & 4 & 0.85 & 0.9 \\
MKW4 & 4 & 0.73 & 0.6 \\
A2255 & 4 & 1.16 & 2.4 \\
A2063 & 5 & 0.86 & 0.2 \\
A3581 & 5 & 1.04 & 0.4 \\
\hline
\end{tabular}

each photon within a circular region of $0.3^{\circ}$ radius centered on A0119, A3112, A4038, A0400, 2A0335, A1367, MKW4, A2255, A2063, and A3581. Note that we excluded ZwIII54 from the sample shown in Table 3, because the probability, $10.4 \%$, of obtaining the observed number of counts from background emission by chance for this galaxy cluster is significantly higher than that for other galaxy clusters from Table 3. We used the nearestneighbor method to test the homogeneity hypothesis and calculate the sum of the distances between each event and its nearest neighbor in time. The nearest-neighbor method was initially introduced by Skellam (1952), where the ratio of the expected and observed mean value of the nearest-neighbor distances is used to determine whether a data set is clustered. This provides a test statistic that requires no partitioning scheme. Note that the observed number of counts in a short time-interval depends on exposure duration. The exposure duration for successive intervals of time corresponding to the precession period of the orbit of the Fermi spacecraft, $\approx 55$ days, is roughly constant. Since we used the 52.5 months of the data, the observation time contains $\approx 28$ time intervals of almost equal exposure. Performing Monte Carlo simulations for the homogeneous distribution of counts in time, we calculated the number of simulation runs with the statistic lower than that calculated for each galaxy cluster from Table 3 . The obtained number of simulation runs with the statistic lower than that for A0199 is $12 \%$ of the simulations, A3112
$-6 \%$, A4038 -77\%, A0400 -54\%, 2A0335-48\%, A1367 $-60 \%$, MKW4 -57\%, A2255-17\%, A2063-8\%, and A3581 $-17 \%$. We also performed a more powerful test for homogeneity by calculating the probability of several independent simulation runs with a given number of simulated counts. From this we obtained the statistic in each simulation run that was lower than that which was derived from the observation of one of the galaxy clusters whose number of counts in its direction was the same as the simulated number. The probability that one of three independent Monte Carlo simulation runs with a homogeneous distribution of three photons in time gives the statistic lower than that for A0199, and two other simulation runs give the statistics lower than those for A3112 and A4038, equals 2.2\%. The probability that one of five independent Monte Carlo simulation runs with a homogeneous distribution of four photons in time gives the statistic lower than that for A0400, and four other simulation runs give the statistics lower than those for $2 \mathrm{~A} 0335$, A1367, MKW4, and A2255, is lower than 6.3\%. The probability that one of two independent Monte Carlo simulation runs with a homogeneous distribution of five photons in time gives the statistic lower than that for A2063, and the other simulation run gives the statistic lower than that for A3581, equals $2.0 \%$. We conclude that the observed gamma rays are not homogeneously distributed in time and that the observed gamma-ray excess towards the selected galaxy clusters cannot be entirely produced by the emission via neutral pion decay and by the IC emission.

Before starting the search for time-variable gamma-ray sources in the directions of the selected galaxy clusters, we note that different sky-survey profiles were used on Fermi and, therefore, the on-source exposure also varies in time because of the use of different sky-survey profiles. To include the exposure variation due to the sky-survey profiles in the present analysis, we first calculated the exposure profile for each of ten regions and the count rates within the regions with a radius of $4^{\circ}$ centered on the galaxy clusters as a function of time. Both the exposure profile and count rate have a temporal feature corresponding to change from the sky survey with a rocking angle of $35^{\circ}$ to the sky survey with a rocking angle of $50^{\circ}$ on 3 September 2009. This temporal feature is present for eight of these ten sky regions. The exposure is almost uniform in time for Abell 3112 and Abell 4038. The average exposure over a period of time before 3 September 2009 is higher by $\approx 25 \%$ than that after 3 September 2009 for seven of the ten sky regions. Note that the average exposure over a period of time before 3 September 2009 is shorter by $\approx 20 \%$ than that after 3 September 2009 for Abell 2255, which is situated in the vicinity of the north celestial pole. To take this effect into account, we distributed the observed number of photons in the direction of each of the ten galaxy clusters according to their exposure profiles. This was made by using the function inversion method. We calculated the cumulative exposure distribution function $\mathrm{F}(\mathrm{x})$ and generated a random number, $q$, which is distributed uniformly on the interval, and then solved the equation, $F(x)=q$. A random number $x$ was distributed in the interval with the probability distribution proportional to the exposure time profile. By performing the Monte Carlo simulations and applying the nearest-neighbor test statistic, we found that the results presented above agree well with those obtained by taking the calculated exposure profiles into account.

In Sect. 3.3, we study the catalog of AGNs to search for time-variable gamma-ray sources towards the selected galaxy clusters. 
Table 4. List of galaxy clusters that are possibly contaminated by CGRaBS sources, located at a projected distance $<0.6^{\circ}$ from the cluster centers.

\begin{tabular}{lcllc}
\hline \hline $\begin{array}{l}\text { Cluster } \\
\text { name }\end{array}$ & $\begin{array}{c}\text { Number of } \\
\text { counts }\end{array}$ & $\begin{array}{l}\text { Redshift of } \\
\text { a cluster }\end{array}$ & $\begin{array}{l}\text { Redshift of } \\
\text { a blazar }\end{array}$ & $\begin{array}{c}\text { Max. energy } \\
(\mathrm{GeV})\end{array}$ \\
\hline A0400 & 4 & 0.024 & 1.381 & 50.8 \\
A3395s & 1 & 0.0498 & 2.051 & 43.5 \\
A3558 & 1 & 0.048 & 1.326 & 19.7 \\
A3581 & 5 & 0.0214 & 2.43 & 16.8 \\
A2029 & 2 & 0.0767 & 0.084 & 21.4 \\
A2147 & 0 & 0.0351 & 0.109 & - \\
A2634 & 1 & 0.0312 & 0.372 & 15.1 \\
A2657 & 2 & 0.0404 & 0.667 & 24.4 \\
\hline
\end{tabular}

\subsection{Time variable sources towards galaxy clusters}

Many of the high-latitude 2FGL gamma-ray sources are associated with the bright, flat radio-spectrum AGNs known as blazars. A uniform all-sky Candidate Gamma-Ray Blazar Survey (CGRaBS) selected primarily by flat radio spectra was conducted by Healey et al. (2008) to assemble a large catalog of possible gamma-ray AGNs suitable for identification of high-latitude gamma-ray sources detected with Fermi-LAT. The CGRaBS catalog contains 1625 gamma-ray candidate sources with radio and X-ray properties similar to those of the EGRET blazars, spread uniformly across the $|b|>10^{\circ}$ sky. Eighty-one per cent of these gamma-ray candidate sources have measured redshifts. Identification of a fraction of gamma-ray sources towards the 55 selected galaxy clusters with blazars will allow us to focus on the gamma rays incoming from galaxy clusters. Note that not all blazars detected by Fermi-LAT are included in the CGRaBS catalog and that $>1000$ sources from the CGRaBS catalog have not yet been detected by Fermi-LAT.

We searched for gamma-ray candidate sources from the CGRaBS catalog within a circle of $0.6^{\circ}$ (which slightly exceeds the mean virial radius and the 2 PSF) towards the selected galaxy clusters and found that gamma-ray candidate sources are present towards eight galaxy clusters, A0400, A3395s, A3558, A3581, A2029, A2147, A2634, and A2637. The distances between each of these galaxy clusters and its nearest gamma-ray source candidate from the CGRaBS catalog is 26.6' for A0400, $9.9^{\prime}$ for $\mathrm{A} 3395 \mathrm{~s}, 20.2^{\prime}$ for $\mathrm{A} 3558,30.8^{\prime}$ for A3581, 28.8' for A2029, 20.2' for A2147, 33.3' for A2634, and 31.3' for A2657. We list the redshifts of these galaxy clusters and of the nearest gamma-ray CGRaBS source for each of these clusters, and the number of counts within a circle of $0.3^{\circ}$ radius around each of these galaxy clusters in Table 4. This table shows that six of these eight blazars have redshifts higher than 0.35 . Note that two of these galaxy clusters, A0400 and A3581, are also present in Table 3. The associated CGRaBS gamma-ray candidate sources with A0400 and A3581 are very distant and have redshifts of 1.381 and 2.43. Note that CGRaBs sources are gamma-ray candidate sources and, therefore, the probability of detecting a signal from CGRaBs sources is expected to be higher than that from an arbitrary position in the sky. Two of these eight CGRaBs sources have previously been associated with gamma-ray sources, namely CGRaBS J0625-5438 towards Abell 3395s (1FGL J0625.9-5430 in the LAT one-year point source catalog) and CGRaBS J1603+1554 towards Abell 2147 (3EG J1603+1554 in the third EGRET catalog). The absence of these two sources in the 2 FGL catalog suggests possible time variablity of their fluxes.
We calculated the number of CGRaBS candidate gamma-ray sources within the first sixteen annuli with a surface of $\pi\left(0.6^{\circ}\right)^{2}$ centered on the 55 selected galaxy clusters and found that the first inner annulus contains eight CGRaBS sources while the mean value of CGRaBS sources within an annulus is 2.8. Thus, the probability of the presence of $\geq 8$ CGRaBS sources within the innermost annulus is only $\approx 0.8 \%$. We also calculated the number of CGRaBS candidate gamma-ray sources within the first sixteen annuli with a surface of $\pi\left(0.5^{\circ}\right)^{2}$ (the radius of the first annulus corresponds to the mean virial radius of the 55 selected clusters) and found that each of the first two annuli contains four CGRaBS sources. The probability of the presence of $\geq 4$ CGRaBS sources within this annulus is $\approx 13.0 \%$. We conclude that the number of CGRaBS gamma-ray candidate sources towards the selected galaxy clusters exceeds the expected number within the central annulus with a radius of $0.5^{\circ}$ (and $0.6^{\circ}$ ). A possible explanation of the high concentration of CGRaBS sources towards these galaxy clusters is a statistical deviation from the expectation.

Note that high-energy gamma rays interact with longwavelength photons of the extragalactic background light and produce electron-positron pairs (the Breit-Wheeler process), see, for example, Kneiske et al. (2002); Franceschini et al. (2008); Finke et al. (2010). The absoption of gamma rays from distant sources, such as blazars, via $\mathrm{e}^{-}-\mathrm{e}^{+}$pair creation leads to a decrease of the observed flux at high energies, $E_{\gamma}>20 \mathrm{GeV}$ (e.g., Finke et al. 2010). We used the absoption optical depth of gamma-ray photons as a function of observed gamma-ray photon energy, calculated by Finke et al. (2010), to test the possibility of observing high-energy photons from the distant CGRaBS sources at $z=1.381,2.051,1.326$, and 2.43, towards A0400, A3395s, A3558, and A3581, respectively. We list the highest observed energy of photons towards these galaxy clusters in Table 4. We take the values of the absoption optical depth from the tables ${ }^{3}$. The absorption optical depth for a gamma-ray with observed energy of $50.8 \mathrm{GeV}$ emitted at $z=1.38$ is $\approx 0.57$, for a gamma-ray with observed energy of $43.5 \mathrm{GeV}$ emitted at $z=2.05$ is $\approx 0.77$, for a gamma-ray with observed energy of $19.7 \mathrm{GeV}$ emitted at $z=1.33$ is $\approx 0.022$, and for a gamma-ray with observed energy of $16.8 \mathrm{GeV}$ emitted at $z=2.43$ is $\approx 0.1$. We calculated the probability of detecting high-energy gamma rays towards these CGRaBS sources in the framework of the absorption model of gamma rays on the extragalactic backround light and found that the detection of the highest energy gamma rays towards the majority of these CGRaBS sources is consistent with the expectation from the absorption model. However, the probability of detecting a $43.5 \mathrm{GeV}$ photon towards A3395s without observing any photons with energies between $10 \mathrm{GeV}$ and $43.5 \mathrm{GeV}$ towards this cluster is only $0.25 \%$ and, therefore, it is hardly possible that the detected $43.3 \mathrm{GeV}$ photon was emitted by a distant blazar.

\subsection{Likelihood analysis of gamma-ray emission in the directions of A0400 and A3581}

We performed a binned likelihood analysis of gamma-ray emission at the positions of the Abell 400 (A0400) and Abell 3581 (A3581) galaxy clusters. These two clusters were selected for analysis because of their presence both in Table 3 (clusters with the largest number of events) at $E>10 \mathrm{GeV}$ and in Table 4 (clusters that are possibly contaminated by CGRaBS candidate gamma-ray sources). The presence of possible gamma-ray

http://www.phy.ohiou.edu/ finke/EBL/ 
sources, namely a binary black hole system (3C 75) in A0400 and a blazar (PKS 1404-267) in A3581, also provides a good reason for searching for gamma-ray emission from these galaxy clusters. The test statistic (TS) was employed to evaluate the significance of the gamma-ray fluxes coming from these two galaxy clusters. The TS value was used to assess the goodness of fit and is defined as twice the difference between the log-likelihood functions maximized by adjusting all parameters of the model, with and without the source, and under the assumption of a precise knowledge of the Galactic and extragalactic diffuse emission. We used the publicly available tool (gtlike), released by the Fermi-LAT collaboration, to perform a binned likelihood analysis. For each analyzed source, we selected events with energy $E>100 \mathrm{MeV}$ in a circular region of interest of $20^{\circ}$ radius. We used the first 52.5 months of the Fermi-LAT data and the good time intervals such that the region of interest does not reach below the Earth limb. We used the spectral-spatial templates, gal_2yearp7v6_v0.fits and iso_p7v6source.txt, for the Galactic and extragalactic diffuse emission, respectively. To produce a spectral-spatial model for each galaxy cluster, we fixed their positions at the localization of 3C 75 for A0400 and of PKS 1404267 in A3581 and included the gamma-ray sources from the 2FGL catalog. The spectra of 2FGL sources were taken from the 2FGL catalog. The spectral parameters of most of the 2FGL sources were fixed at the 2FGL values, while the parameters of the strongest sources in the region of interest were left free in the likelihood fit. We modeled the emission from these two galaxy clusters under the assumption of a point source with a power-law spectral distribution. The likelihood ratio test shows that the TS values for A0400 and A3581 equal 41.7 and 5.3. The approximate value of the significance of detection is given by $\sqrt{T S} \sigma$ and, therefore, the significance of detection is $6.4 \sigma$ and $2.3 \sigma$ for A0400 and A3581. Note that the possible explanation of a higher significance value for A400 is that diffuse emission is strongly inhomogeneous on a scale of $\simeq 5^{\circ}$ towards this galaxy cluster. The performed likelihood analysis shows that the gamma-ray sources towards these galaxy clusters are promising targets for an analysis in the future.

To check whether the CGRaBS sources located within the circles with a radius of $0.6^{\circ}$ centered on A0400 and A3581 can strongly contaminate the gamma-ray signal observed from these two galaxy clusters, we first compared the number of counts with energies $>10 \mathrm{GeV}$ within the circles with a radius of $0.3^{\circ}$ centered on the position of each of these galaxy clusters with those within the circles centered on the position of these CGRaBS sources. The numbers of counts within the circles with a radius of $0.3^{\circ}$ centered on the position of $\mathrm{A} 0400$ and on the position of CGRaBS J0258+0541 are 4 and 3. The numbers of counts within the circles with a radius of $0.3^{\circ}$ centered on the position of A3581 and on the position of CGRaBS J1409-2657 are 5 and 0. This analysis shows that the signal from A0400 can be contaminated by CGRaBS J0258+0541 and that CGRaBS J1409-2657 does not contribute to the signal in the direction of A3581. To confirm this result, we performed the likelihood analysis again and changed the position of a tentative gamma-ray source from the galaxy cluster location to the CGRaBS source position. We found that the model with a gamma-ray source at the position of CGRaBS J0258+0541 is less probable by a factor of 2 than the model with a gamma-ray source at the position of the center of A0400. The model with a gamma-ray source at the position of CGRaBS J1409-2657 is less probable by a factor of 14 than the model with a gamma-ray source at the position of the center of A3581. Therefore we conclude that the association of the gamma-ray signal in the direction of A0400 with CGRaBS
$\mathrm{J} 0258+0541$ is probable and that the gamma-ray signal from A3581 is not contaminated by CGRaBS J1409-2657.

\subsection{Cool-core and non-cool core galaxy clusters}

Clusters of galaxies with dense gaseous cores have central cooling times much shorter than a Hubble time. XMM-Newton observations of cool-core galaxy clusters have shown that the spectral features predicted by the cooling-flow models are absent from the X-ray spectra (Peterson et al. 2001, 2003). The likely source of energy that compensates for gas cooling losses comes from the central AGN (e.g., Churazov et al. 2000; David et al. 2001; Böhringer et al. 2002). The dense gaseous cores in coolcore clusters can lead to an increase in gamma-ray emission due to neutral pion decay produced in the collisions of relativisitic protons with protons of the ICM, while the central AGN itself can also be a source of gamma-ray emission. The probability of finding a cluster center radio AGN increases from $\sim 45 \%$ to $\sim 100 \%$ for non-cool core and strong cool-core clusters (Mittal et al. 2009). Thus, a gamma-ray signal from cool-core clusters higher than that from non-cool core clusters can possibly be interpreted as evidence for emission of central AGNs. Non-cool core clusters in turn often show evidence of a merger and, therefore, CR hadrons and electrons accelerated in merger shocks can lead to gamma-ray emission via the neutral pion decay or IC effect. In this section we analyze a sample of cool-core galaxy clusters and a sample of non-cool core clusters to study gammaray emission of these two distinct types of galaxy clusters.

We divided the 55 selected galaxy clusters into the two samples using the definition from Chen et al. (2007). If the ratio of the mass deposition rate $\dot{M}$ to the cluster mass $M_{500}$ for a galaxy cluster exceeds $10^{-13} \mathrm{yr}^{-1}$, the galaxy cluster belongs to the sample of cool-core clusters. The second sample of galaxy clusters consists of galaxy clusters with a ratio $\dot{M} / M_{500}$, lower than $10^{-13} \mathrm{yr}^{-1}$. We took the values of $\dot{M} / M_{500}$ and $M_{500}$ from Tables 1 and 4 of Chen et al. (2007). There are two galaxy clusters, A1651 and A2063, with calculated ratios of $\dot{M} / M_{500}$ close to $10^{-13} \mathrm{yr}^{-1}$. We included these two clusters in the sample of cool-core clusters. The sample of cool-core clusters consists of 27 clusters and that of non-cool core clusters consists of 28 clusters.

Using the spectral-spatial templates presented above, we calculated the significance of detection of a gamma-ray signal from the two samples. The method used to evaluate the statistical significance is analogous to that applied in Sect. 3.1. The evaluated values of the significance of detection are shown in Table 5. We found that the significance of a gamma-ray signal from the sample of cool-core clusters, $\simeq 5 \sigma$, is significantly higher than that from the sample of non-cool core clusters, $\simeq 2 \sigma$. Furthermore, we conclude that the observed excess of gamma-ray emission in the direction of the 55 selected clusters is mainly owing to gamma rays coming from the cool-core clusters.

Assuming that the numbers of observed photons in the two samples of galaxy clusters can be modeled by a Poisson distribution, we determined whether the two Poisson samples have the same mean. For this analysis, we used the test-statistics, $T S=\left(O_{1}-E_{1}\right)^{2} / E_{1}+\left(O_{2}-E_{2}\right)^{2} / E_{2}$, where $O_{1,2}$ are the observed number of photons within a circle of $0.3^{\circ}$ centered on galaxy clusters from each sample and $E_{1,2}$ is the number of photons expected under the assumption that the two Poisson samples have the same mean. We found that the probability that both samples have the same mean is $4.6 \%$. Therefore, the gammaray properties of the cool-core clusters significantly differ from those of the non-cool core clusters. Since the contribution of 
Table 5. Significance of signal detection for the samples of cool-core and non-cool core galaxy clusters.

\begin{tabular}{lcc}
\hline \hline Template & Cool-core clusters & Non-cool core clusters \\
\hline point source & $4.3 \sigma$ & $1.8 \sigma$ \\
disk $\left(R=0.15^{\circ}\right)$ & $4.9 \sigma$ & $2.0 \sigma$ \\
disk $\left(R=0.25^{\circ}\right)$ & $5.0 \sigma$ & $2.0 \sigma$ \\
disk $\left(R=0.375^{\circ}\right)$ & $4.6 \sigma$ & $1.8 \sigma$ \\
disk $\left(R=0.5^{\circ}\right)$ & $3.5 \sigma$ & $1.9 \sigma$ \\
\hline
\end{tabular}

gamma rays from CGRaBS sources to the signal in the directions of galaxy clusters can be significant, we repeated the analyses of cool-core and non-cool core galaxy clusters and excluded the galaxy clusters with CGRaBS sources within a circle of $0.6^{\circ}$ from the samples of cool-core and non-cool core clusters (note that we included Abell 3581 in the reduced sample of cool-core clusters because a close CGRaBS source cannot contribute to the emission in the direction of this galaxy cluster, see Sect. 3.4). We found that the probability that the two reduced samples have the same mean is $5.1 \%$. We conclude that the contribution of cool-core clusters to the gamma-ray emission from the 55 selected clusters dominantes that of non-cool core clusters.

Note that our selection of 55 galaxy clusters is based on the flux-limited cluster sample from the HIFGLUGS catalog. Therefore, the expected proportionality between X-ray and neutral pion decay induced gamma-ray fluxes (see Ensslin et al. 1997) leads to a testable prediction about higher gamma-ray fluxes from brighter X-ray galaxy clusters. Taking this prediction into account, we performed a likelihood analysis and weighted the observed number of gamma rays from each galaxy cluster with its expected gamma-ray flux. We calculated the Poisson log-likelihood for each of the spectral-spatial models using two weighting techniques: 1) combining the observed count maps with equal weights (as in Sect. 3.1); and 2) weighting the observed number of counts with expected gamma-ray fluxes. We computed the difference in the maximized values of the loglikelihood $(L)$ functions for both weighting techniques and for each spectral-spatial model and found that the maximum likelihood for the "equal weights" model is higher than that for the hadronic model of gamma-emission. We used the Akaike information criterion to measure the relative quality of the models that support these two weighting techniques and found that the hadronic model of gamma-emission is $\exp \left(L_{2}-L_{1}\right) \simeq 5 \times$ $10^{-3}$ times as probable as the "equal weights" model to minimize the information loss. The significance of the detection of a signal in the direction of the 55 selected galaxy clusters is in the range of $2.1-2.7 \sigma$ (depending on the spectral-spatial models) when the weighting technique assuming the expected gammaray fluxes from the hadronic model is used. Therefore, there is no convincing evidence that the observed signal is due to neutral pion decay, at least in the simplest version of the model assuming $F_{\gamma} \propto F_{X}$.

\subsection{Upper limit on the ratio of relativistic hadron-to-thermal energy density}

We re-evaluated the significance of a gamma-ray signal from clusters of galaxies by withdrawing eight galaxy clusters that are possibly contaminated by CGRaBS gamma-ray candidate sources (see Sect. 3.3) from our sample. Using the method described in Sect. 3.1 and the templates for gamma-ray emission produced above, we found that the values of the significance level are $3.3 \sigma, 3.6 \sigma, 3.2 \sigma$, and $2.3 \sigma$ for the disk templates with radii of $0.15^{\circ}, 0.25^{\circ}, 0.375^{\circ}$, and $0.5^{\circ}$, respectively. The significance level for the point source template is $2.7 \sigma$. For the ring-like model, we found no evidence for an excess over background. Note that the mean number of counts within a circle with a radius of $0.3^{\circ}$ centered on the galaxy clusters possibly associated with CGRaBS sources is $2.0 \pm 0.5$, while the corresponding average number calculated for the 47 selected galaxy clusters (excluding those that have the possible association with CGRaBS sources) is $1.6 \pm 0.18$. For comparison, the mean background contribution to the same region is $\simeq 0.96$ per cluster. A slightly lower flux in the reduced sample is yet another reason (in addition to the smaller sample size) why the detection significance is lower in the reduced sample.

The energy density stored in hadronic cosmic rays (CR) relative to the thermal energy density of the ICM gas can be constrained by using the observed gamma-ray and X-ray fluxes from galaxy clusters (Ensslin et al. 1997; Ackermann et al. 2010a). Using the Fermi Science Tools ${ }^{2}$, we calculated the exposure weighted by the power-law with a photon index of -2.1 for each of the 47 galaxy clusters to convert count numbers into gammaray fluxes at $E>10 \mathrm{GeV}$. Note that the calculated exposure values are in the range of $(130-200) \times 10^{9} \mathrm{~cm}^{2} \mathrm{~s}$. The mean is $144 \times 10^{9} \mathrm{~cm}^{2} \mathrm{~s}$ and the standard deviation is $13 \times 10^{9} \mathrm{~cm}^{2} \mathrm{~s}$. The highest exposure values are for Abell 2255 and Abell 2256 and equal to $192 \times 10^{9}$ and $198 \times 10^{9} \mathrm{~cm}^{2} \mathrm{~s}$. These two galaxy clusters are located in the vicinity of the north celestial pole where the exposure of Fermi-LAT is highest. We took the X-ray fluxes from these galaxy clusters and their plasma temperatures from the catalogs published by David et al. (1993); Reiprich \& Böhringer (2002). We took the production rate of gamma rays from Drury et al. (1994) for a spectral index, $s=4.1$, of the parent CR hadron distribution $\mathrm{d} N \propto p^{-s} \mathrm{~d}^{3} p$, where $p$ is the momentum, and extrapolated the production rate at $E>1 \mathrm{TeV}$ to $E>10 \mathrm{GeV}$. Note that the production rate of gamma rays is defined as the gamma-ray emissivity normalized to the CR hadron energy density. Using Eq. (10) from Ensslin et al. (1997) and the calculated production rate of gamma rays at $E>10 \mathrm{GeV}$, we obtained the relation between the observed fluxes of gamma rays, $F_{\gamma}(>10 \mathrm{GeV})$, and $\mathrm{X}$-rays, $F_{\mathrm{X}}$, that is given by

$$
\frac{F_{\gamma}(>10 \mathrm{GeV})}{F_{\mathrm{X}} / \mathrm{erg}} \approx 1.33\left(\frac{k_{\mathrm{B}} T}{\mathrm{keV}}\right)^{1 / 2} \alpha,
$$

where $\alpha$ is the scaling ratio between the thermal $\epsilon_{\mathrm{th}}$ and $\mathrm{CR}$ hadron energy $\epsilon_{\mathrm{CR}}$ densities, $\epsilon_{\mathrm{CR}}=\alpha \times \epsilon_{\mathrm{th}}$. Note that the gamma production rate may be taken to be proportional to the number density of relativistic hadrons (Drury et al. 1994) and that the energy density of relativistic particles only slightly depends on the low-energy bound for hard power-law hadron spectra with $s=4.1$. Moreover, soft power-law hadron spectra, e.g. $s=4.7$, produce softer gamma-ray spectra and the value of CR hadron energy density derived by means of this method depends on the assumed power-law hadron spectra. For softer hadron spectra, the factor of 1.33 in Eq. (5) will be lower (e.g., $\simeq 0.08$ for $s=4.7$ ) and the contribution of hadrons of energy below about $1 \mathrm{GeV}$ per nucleon, which do not generate observable gamma rays, to the total energy density will dominate.

To estimate the scaling ratio $\alpha$ from the observed gammaray fluxes in the directions of galaxy clusters, we performed a likelihood analysis. Using Eq. (5) and the calculated exposure values, we derived the expected number of counts produced via 
neutral pion decay emission assuming the same scaling ratio $\alpha$ for each of these 47 galaxy clusters. The number of counts from background emission for each galaxy cluster was calculated by using a region of interest of $4^{\circ}$ around each cluster excluding the 2FGL sources and the possible central gamma-ray source. Using the number of observed counts, we calculated the joint likelihood for different values of the scaling ratio $\alpha$. We found that the best-fit value of the ratio of the relativistic hadron to thermal energy density is $1 \%$, while the $95 \%$ confidence inverval is $[0.3 \%, 2 \%]$.

We also calculated the $95 \%$ upper limits on the ratio of the relativistic hadron to thermal energy density assuming a softer power-law hadron spectrum with $s=4.2,4.3$, and 4.4 and using the production rates of gamma rays from Table 1 of Drury et al. (1994). We found that the $95 \%$ upper limits are $\simeq 3 \%, \simeq 4 \%$, and $\simeq 6 \%$, respectively.

The assumption that this gamma-ray signal is caused by sources that are variable in time (AGNs) allows us to put an upper limit on the ratio of the relativistic hadron to thermal energy density equal to $2 \%$, assuming a power-law hadron spectrum with $s=4.1$. The possible relativistic hadron component with an energy density of $2 \%$ of that of the thermal plasma does not violate the constraints obtained by an alternative method by Churazov et al. (2008) and its presence needs to be tested by a combined analysis of the Fermi-LAT observations towards the extended galaxy clusters with high X-ray fluxes, such as Virgo, Perseus, Fornax, Hydra, Coma, and Centaurus. An analysis of these extended galaxy clusters is beyond the scope of our paper and requires a more sophisticated technique to deal with the spatial extension of these nearby X-ray brightest clusters of galaxies.

\section{Spectral analysis in three energy bands}

In the previous sections we presented the analysis of gammaray emission towards 55 galaxy clusters at energies greater $10 \mathrm{GeV}$. The tentative gamma-ray excess towards these galaxy clusters at $E>10 \mathrm{GeV}$ was detected. To validate the presence of this gamma-ray excess, below we analyze gamma-ray emission towards these galaxy clusters in several energy bands. We chose three energy bands for our spectral analysis, which are 3333-5773 MeV, 5773-10000 MeV, and >10 GeV. Low boundaries of these three energy bands are logarithmically spaced and the low-energy boundary of the first band was chosen taking into account that the high spatial resolution at $E \gtrsim 3 \mathrm{GeV}$ is sufficient to avoid contamination of a signal from galaxy clusters by gamma rays incoming from 2FGL sources.

We used the point and disk source models described in Sect. 3.1 to calculate the number of observed photons towards galaxy clusters in the three energy bands 3333-5773 MeV, 5773-10000 MeV, and >10 GeV. To perform the spatial analysis, we binned the count maps into concentric annuli with a surface area of $\pi \times \mathrm{PSF}^{2} / 2$. Note that the number of background photons within the region of interest of $4^{\circ}$ centered on the location of each galaxy cluster is $\gtrsim 100$ in each of the three energy bands. To calculate the number of background photons in each regions, we masked the 2FGL sources within the region of interest and corrected the obtained number of background photons taking into account the masked surface area. We also took the variation of background emission over the sky into account by applying the following procedure: we calculated both the number of counts $s$ observed from the gamma-ray source and the uncertainty due to background emission $\sigma$ for each region of
Table 6. Evaluated number of counts from the source in the direction of galaxy clusters in three energy bands.

\begin{tabular}{lcll}
\hline \hline Template & $N_{\text {counts }}$, band1 & $N_{\text {counts }}$, band2 & $N_{\text {counts }}$, band3 \\
\hline point source & $18.6 \pm 16.0$ & $23.9 \pm 7.5$ & $28.8 \pm 6.5$ \\
disk $\left(R=0.15^{\circ}\right)$ & $21.3 \pm 17.3$ & $26.1 \pm 8.1$ & $41.5 \pm 8.3$ \\
disk $\left(R=0.25^{\circ}\right)$ & $25.1 \pm 18.9$ & $29.2 \pm 9.0$ & $49.1 \pm 9.4$ \\
disk $\left(R=0.375^{\circ}\right)$ & $33.4 \pm 22.0$ & $34.0 \pm 10.7$ & $55.4 \pm 11.7$ \\
disk $\left(R=0.5^{\circ}\right)$ & $44.4 \pm 25.4$ & $37.4 \pm 12.6$ & $54.2 \pm 13.8$ \\
\hline
\end{tabular}

interest in each energy band using the procedure described in Sect. 3.1. Since the background emission varies from one region of interest to another, we calculated the weighted mean of the number of counts observed from the gamma-ray source $s_{\mu}$ and the variance of the mean $\sigma_{\mu}^{2}$ in each energy band, as $s_{\mu}=\sum_{k}\left(s_{k} / \sigma_{k}^{2}\right) / \sum_{k}\left(1 / \sigma_{k}^{2}\right)$ and $\sigma_{\mu}^{2}=1 / \sum_{k}\left(1 / \sigma_{k}^{2}\right)$, where $k$ is the the index of summation referring to galaxy clusters.

The evaluated numbers of photons from the source in the directions of galaxy clusters in each energy band are shown in Table 6. The energy bands are enumerated in increasing order in this table. To calculate the PSF in each energy band, we adopted the power-law spectral distribution for each template with a photon index of 2.1. Table 6 shows that the number of photons in the third energy band, that is, $5773-10000 \mathrm{MeV}$, is greater than zero at a statistical level of $\approx 3 \sigma$. Thus, the second and third energy bands provide an evidence for the possible gamma-ray signal from galaxy clusters. Using a likelihood analysis, we calculated the best-fit value for the photon index and the $95 \%$ confidence level interval for the observed photon index. We found that the best-fit photon index is 1.7, providing 23.3, 15.9, and 29.9 photons in the energy bands of 3333-5773 MeV, $5773 \mathrm{MeV}-10 \mathrm{GeV}$, and >10 GeV, respectively, for the point source template. The $95 \%$ confidence interval for the photon index is $[1.6,1.9]$ and, therefore, the observed photon index is harder than that expected for neutral pion decay emission.

We estimated the significance of a gamma-ray signal towards galaxy clusters by using the likelihood ratio method (e.g., see $\mathrm{Li} \& \mathrm{Ma} 1983)$. In the present problem there is one unknown parameter - the expected number of source photons. Note that the expected number of background photons is derived from outer spatial bins where the number of photons coming from the source is negligible. The statistical hypothesis tested here is that no extra source exists and all observed photons are due to background. If the errors are Gaussian, the likelihood is given by $\mathcal{L}=\exp \left(-\chi^{2} / 2\right)$, so that minimizing $\chi^{2}$ is equivalent to maximizing $\mathcal{L}$. The correctness of the estimation procedure follows from the fact that the variable $-2 \ln \left(\mathcal{L} / \mathcal{L}_{\max }\right)$ is distributed like $\chi^{2}$ with one degree of freedom if there is one parameter of the problem (see Wilks 1962). We re-evaluated the significance of the gamma-ray excess at energies $>10 \mathrm{GeV}$ and confirm the results presented in Table 1 of Sect. 3.1, which were obtained by means of an alternative method. We performed a combined likelihood analysis in the three energy bins using the best-fit photon index of 1.7. We found that the value of the significance level is $5.4 \sigma$ for the point source template (cf. Table 1) and that the values of the significance level are $5.6 \sigma, 6.0 \sigma, 5.6 \sigma$, and $5.1 \sigma$ for the templates describing gammaray emission from disks with radii of $0.15^{\circ}, 0.25^{\circ}, 0.375^{\circ}$, and $0.5^{\circ}$, respectively. Thus, including the three low-energy bands in the analysis increases the significance of detection 
of a tentative gamma-ray excess in the directions of galaxy clusters.

\section{Conclusions}

Clusters of galaxies are promising targets for gamma-ray telescopes. Gamma rays can be emitted from the ICM via the decay of neutral pions produced in inelastic proton-proton collisions or via IC radiation from the interaction of very energetic electrons with $\mathrm{CMB}$ photons. In addition to these two radiative processes, gamma rays can also be emitted by the central AGNs in galaxy clusters. We searched for $10 \mathrm{GeV}$ emission from galaxy clusters using the first 52.5 months of the Fermi-LAT data. We performed a combined analysis of the 55 galaxy clusters selected from the HIFLUCGS catalog such that the virial radius of each selected galaxy cluster does not exceed $1^{\circ}$. We selected photon events with energies above $10 \mathrm{GeV}$ that are incoming from the circular regions with a radius of $4^{\circ}$ and centered on these 55 galaxy clusters. The selection of this energy interval was motivated by the high angular resolution of Fermi-LAT at these energies, which permits us to associate the observed gamma rays more precisely with those that are expected to come from galaxy clusters.

To evaluate the significance of a gamma-ray signal in the direction of the 55 galaxy clusters, we produced six spectralspatial templates (see Fig. 4) and fit the models based on these templates to the observed data. The six models included a point source model, four disk models with radii of $0.15^{\circ}, 0.25^{\circ}$, $0.375^{\circ}$, and $0.5^{\circ}$, and a ring model with inner and outer radii of $0.45^{\circ}$ and $0.65^{\circ}$, respectively. We found that the significance of the observed signal exceeds $4 \sigma$ for a point source model and for the first three disk models. The highest evaluated significance is $\simeq 5 \sigma$ and is provided by the model of gamma-ray emission from a disk of $0.25^{\circ}$ radius. Including the two low-energy bands (3333-5773 MeV, and 5773-10000 MeV) in the analysis increased the significance of detection of a tentative gamma-ray excess in the direction of galaxy clusters from $\simeq 5 \sigma$ to $\simeq 6 \sigma$ for the best-fit model. Fitting a disk model to the data improved the likelihood compared with the point-source hypothesis, but did not provide strong evidence for a spatial extension of the observed gamma-ray signal towards the clusters. We found no evidence for gamma-ray emission from the structure formation shocks described by the ring model.

We analyzed a sample of cool-core galaxy clusters and a sample of non-cool core clusters to study gamma-ray emission of these two distinct types of galaxy clusters. To divide the 55 selected galaxy clusters into the two samples, we applied a selection criterion based on the estimated ratio of $\dot{M} / M_{500}$ to our sample of galaxy clusters. The analysis showed that the contribution of cool-core clusters to the gamma-ray emission from the 55 selected clusters dominates that of non-cool core clusters and that the gamma-ray properties of the cool-core clusters significantly differ from those of the non-cool core clusters.

To reveal the origin of the observed gamma-ray excess in the direction of the 55 selected galaxy clusters, we studied independently the signals from each of these galaxy clusters. We found that there are ten galaxy clusters in our sample, such that the probability of obtaining the observed number of photons from each of these clusters within the circular region with a radius of $0.3^{\circ}$ by chance is lower than $5 \%$. We searched for time variability of the signals from these ten galaxy clusters using the nearest-neighbor method to test the homogeneity of incoming photons in time. The time-variability analysis showed that the homogeneity hypothesis is not valid and that a fraction of the observed photons has been emitted by variable gamma-ray sources, such as AGNs.

We searched for gamma-ray candidate sources from the CGRaBS catalog within a circle of $0.6^{\circ}(\approx 2 \mathrm{PSF})$ towards the selected 55 galaxy clusters and found that the gamma-ray candidate sources are present towards eight galaxy clusters. Six of these eight CGRaBS gamma-ray candidate sources are distant blazars with redshifts higher than 0.35 . We estimated the significance of a gamma-ray signal after excluding eight galaxy clusters that are possibly associated with CGRaBS candidate sources. We found that the significance of gamma-ray detection for this subsample is lower than that derived from the sample of 55 galaxy clusters, and that the highest significance level $(3.6 \sigma)$ is also provided by the disk model with a radius of $0.25^{\circ}$. Comparing the observed fluxes with those expected from the neutral pion decay model, we found that the best-fit value of the ratio of the relativistic hadron to thermal energy density equals $\approx 1 \%$ for the spectral index of the parent CR hadron distribution, $d N \propto p^{-s} \mathrm{~d}^{3} p$, with $s=4.1$. Given that AGNs most likely contribute to the observed flux, this value can be treated as an upper limit on the ratio of the relativistic hadron to thermal energy density of $2 \%$, provided that relativistic and thermal components are mixed. Some of the arguments disfavor the interpretation of the $\simeq 5 \sigma$ excess observed in the direction of galaxy clusters as cosmic-ray-induced emission from the ICM. These arguments include that 1) the detection significance is reduced when weighting the clusters according to their X-ray fluxes; 2) the fitted spectrum of the excess is harder than would be expected from hadronic emission; and 3) the observed gamma rays are not homogeneously distributed in time.

After the submission of this paper, the results of a stacking analysis of Fermi-LAT observations performed by two research groups were released (Ackermann et al. 2014; Huber et al. 2013). The authors used the weighting procedure corresponding to the expected contribution from neutral pion decay emission to the signal from the galaxy clusters. The upper limits on the ratio of the relativistic hadron to thermal energy density derived in our paper are consistent with those obtained by these two research groups. Their conclusion that no neutral pion decay emission from the galaxy clusters is detected agrees with our results.

Acknowledgements. We are grateful to the referee for the constructive suggestions that helped us to improve this paper.

\section{Appendix A}

The $\mathrm{S} / \mathrm{N}$ for the $E>10 \mathrm{GeV}$ band to that of each of the energy bands (1924-3333 MeV, 3333-5773 MeV, and $5773 \mathrm{MeV}-10 \mathrm{GeV}$ ) is shown in Figs. A.1-A.3 for various values of photon indices for source and background power-law spectra.

In Table A.1, we list the names of the 2FGL sources that were masked to evalute the significance of the observed signal in the directions of galaxy clusters, the square root of the TS values at $E>10 \mathrm{GeV}$ (from the 2FGL catalog), and the names of galaxy clusters located within a circle of $4^{\circ}$ radius around each of the sources. 
A\&A 567, A93 (2014)

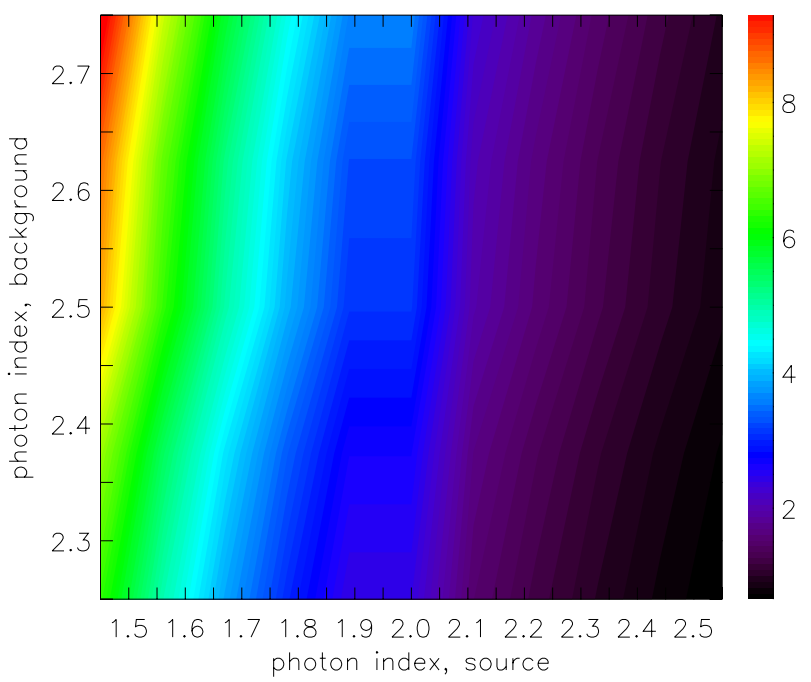

Fig. A.1. Ratio of the $\mathrm{S} / \mathrm{N}$ in the energy band $E>10 \mathrm{GeV}$ to that in the energy band $E=(1924-3333) \mathrm{MeV}$ for various values of photon indices of point source and background power-law spectra.

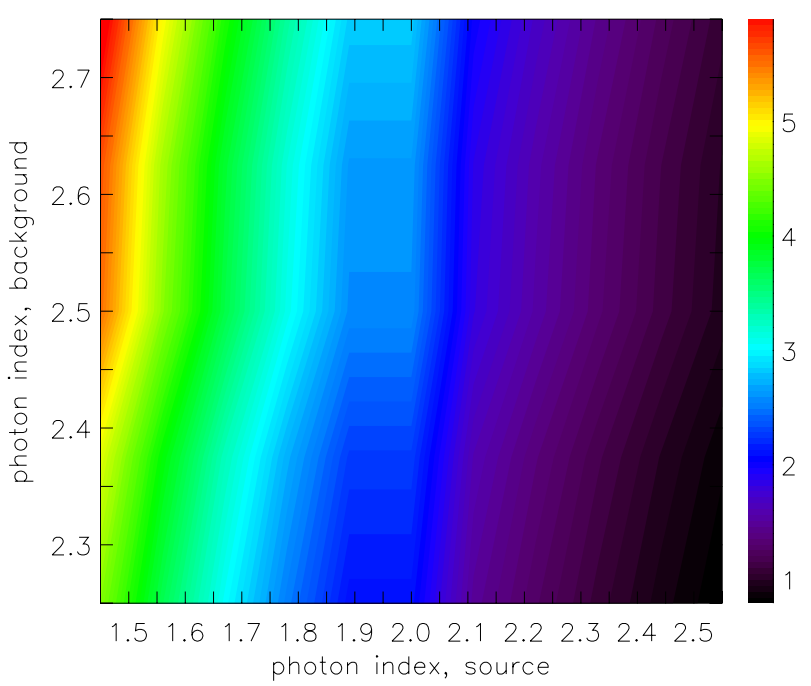

Fig. A.2. Ratio of the $\mathrm{S} / \mathrm{N}$ in the energy band $E>10 \mathrm{GeV}$ to that in the energy band $E=(3333-5773) \mathrm{MeV}$ for various values of photon indices of point source and background power-law spectra.

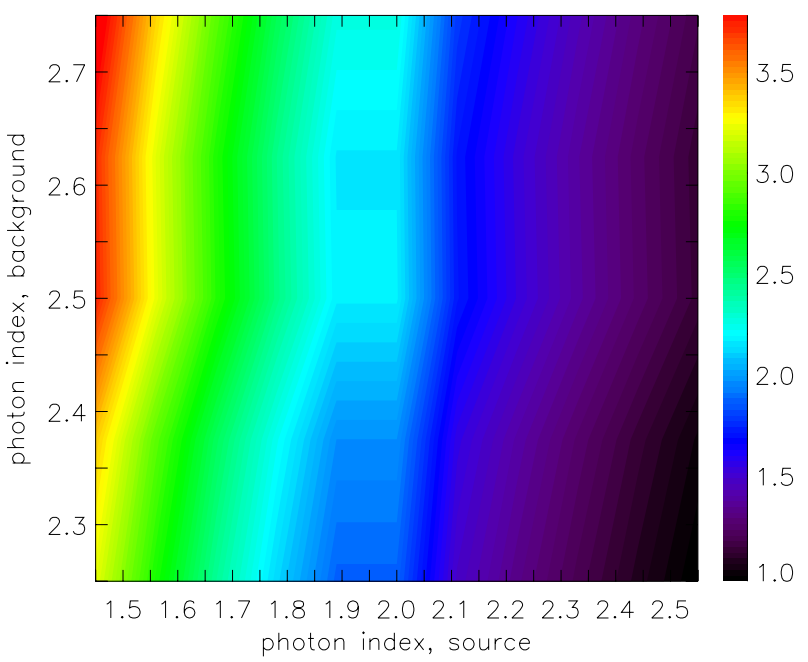

Fig. A.3. Ratio of the $\mathrm{S} / \mathrm{N}$ in the energy band $E>10 \mathrm{GeV}$ to that in the energy band $E=(5773-10000) \mathrm{MeV}$ for various values of photon indices of point source and background power-law spectra.
Table A.1. Masked point sources.

\begin{tabular}{|c|c|c|}
\hline 2GL source name & $\sqrt{T S}$ at $E>10 \mathrm{GeV}$ & Cluster name \\
\hline 2FGLJ0009.9-3206 & 1.7 & A4059 \\
\hline 2FGLJ0050.6-0929 & 7.7 & A0085 \\
\hline 2FGLJ0051.0-0648 & 2.5 & A0085 \\
\hline 2FGLJ0055.0-2454 & 1.4 & A0133 \\
\hline 2FGLJ0056.8-2111 & 3.5 & A0133 \\
\hline 2FGLJ0059.2-0151 & 4.7 & A0119 \\
\hline 2FGLJ0118.8-2142 & 4.0 & A0133 \\
\hline 2FGLJ0112.8+3208 & 3.5 & NGC507 \\
\hline 2 2FGLJ0122.6+3425 & 5.8 & NGC507 \\
\hline 2FGLJ0309.1+1027 & 4.6 & A0399, A0401 \\
\hline 2FGLJ0325.1-5635 & 2.1 & A3158 \\
\hline 2FGLJ0335.3-4501 & 5.7 & A3112 \\
\hline 2FGLJ0338.2+1306 & 5.0 & 2A0335, ZwIII 54 \\
\hline 2FGLJ0414.9-0855 & 3.8 & EXO0422 \\
\hline 2FGLJ0416.8+0105 & 3.4 & NGC1550 \\
\hline 2FGLJ0423.2-0120 & 9.0 & NGC1550 \\
\hline 2FGLJ0424.7+0034 & 3.4 & NGC1550 \\
\hline 2FGLJ0647.7-5132 & 4.0 & A3391 \\
\hline 2FGLJ0710.5+5908 & 10.1 & A0576 \\
\hline 2FGLJ0742.6+5442 & - & A0576 \\
\hline 2FGLJ0856.6-1105 & 6.0 & A0754 \\
\hline 2FGLJ0906.2-0906 & 3.1 & A0754 \\
\hline 2FGLJ1141.9+1550 & 4.2 & A1367 \\
\hline 2FGLJ1154.0-0010 & 7.1 & MKW4 \\
\hline 2FGLJ1229.1+0202 & 4.7 & ZwCl1215 \\
\hline 2FGLJ1256.1-0547 & 16.0 & A1650, A1651 \\
\hline 2FGLJ1310.9+0036 & 5.5 & A1650 \\
\hline 2FGLJ1313.0-0425 & - & A1651 \\
\hline 2FGLJ1315.9-3339 & 4.0 & A3558 \\
\hline 2FGLJ1345.8-3356 & 1.8 & A3562 \\
\hline 2FGLJ1347.0-2956 & 4.2 & A3562, A3571 \\
\hline 2FGLJ1351.1-2749 & 1.3 & A3581 \\
\hline 2FGLJ1406.2-2510 & 5.1 & A3581 \\
\hline 2FGLJ1416.3-2415 & 4.5 & A3581 \\
\hline 2FGLJ1440.9+0611 & 6.1 & MKW8 \\
\hline 2FGLJ1505.1+0324 & - & A2029 \\
\hline 2FGLJ1506.6+0806 & 3.2 & A2029, A2059 \\
\hline 2FGLJ1506.6+0806 & 3.2 & MKW35, A2063 \\
\hline 2FGLJ1508.5+2709 & 3.8 & A2065 \\
\hline 2FGLJ1512.2+0201 & 6.0 & A2029 \\
\hline 2FGLJ1522.1+3144 & 12.8 & A2065 \\
\hline 2FGLJ1539.5+2747 & 2.4 & A2065 \\
\hline 2FGLJ1548.3+1453 & 4.8 & A2147 \\
\hline 2FGLJ1553.5+1255 & 3.5 & A2147 \\
\hline 2FGLJ1607.0+1552 & 1.8 & A2147 \\
\hline 2FGLJ1635.2+3810 & 5.6 & A2199 \\
\hline 2FGLJ1640.7+3945 & 2.8 & A2199 \\
\hline 2FGLJ1642.9+3949 & 2.2 & A2199 \\
\hline 2FGLJ1800.5+7829 & 11.5 & A2256 \\
\hline 2FGLJ1955.0-5639 & 4.0 & A3667 \\
\hline 2FGLJ2317.3-4534 & 2.7 & S1101 \\
\hline 2FGLJ2319.1-4208 & 3.7 & S1101 \\
\hline 2FGLJ2324.7-4042 & 8.5 & S1101 \\
\hline 2FGLJ2327.9-4037 & 0.5 & S1101 \\
\hline 2FGLJ2347.2+0707 & 4.1 & A2657 \\
\hline 2FGLJ2350.2-3002 & 3.1 & A4038 \\
\hline 2FGLJ2353.5-3034 & 4.4 & A4038 \\
\hline 2FGLJ2359.0-3037 & 4.4 & A4038 \\
\hline
\end{tabular}

In Table A.2 we list the arrival time, given in the mission elapse time (MET) units, and the energy for each of the photons within a circular region of $0.3^{\circ}$ radius centered on A0119, A3112, A4038, A0400, 2A0335, A1367, MKW4, A2255, A2063, and A3581. 
D. A. Prokhorov and E. M. Churazov: Counting gamma rays in the directions of galaxy clusters

Table A.2. Photon events within a circular region of $0.3^{\circ}$ radius centered on A0119, A3112, A4038, A0400, 2A0335, A1367, MKW4, A2255, A2063, and A3581.

\begin{tabular}{|c|c|c|}
\hline Cluster name & Arrival time (MET), $\mathrm{s}$ & Energy, GeV \\
\hline A0119 & 261033225 & 10.8 \\
\hline A0119 & 267864212 & 28.3 \\
\hline A0119 & 290446871 & 150.0 \\
\hline A3112 & 247100190 & 66.7 \\
\hline A3112 & 251029235 & 90 \\
\hline A3112 & 268175993 & 11.2 \\
\hline A4038 & 282124076 & 11.9 \\
\hline A4038 & 310302979 & 20.4 \\
\hline A4038 & 369147989 & 13.1 \\
\hline A0400 & 250037714 & 24.1 \\
\hline A0400 & 291576539 & 11.9 \\
\hline A0400 & 297933185 & 50.8 \\
\hline A0400 & 330823308 & 10.7 \\
\hline $2 \mathrm{~A} 0335$ & 283348255 & 11.7 \\
\hline $2 \mathrm{~A} 0335$ & 333270630 & 10.8 \\
\hline $2 \mathrm{~A} 0335$ & 352741781 & 36.8 \\
\hline 2A0335 & 358456858 & 10.3 \\
\hline A1367 & 260628741 & 48.6 \\
\hline A1367 & 314405927 & 14.9 \\
\hline A1367 & 349727501 & 70 \\
\hline A1367 & 351437188 & 22.3 \\
\hline MKW4 & 241698944 & 14.3 \\
\hline MKW4 & 255282333 & 10.3 \\
\hline MKW4 & 263466545 & 10.9 \\
\hline MKW4 & 323071228 & 11.5 \\
\hline A2255 & 327382581 & 60 \\
\hline A2255 & 338658407 & 12.3 \\
\hline A2255 & 361107399 & 14.6 \\
\hline A2255 & 373040091 & 177.5 \\
\hline A2063 & 245663292 & 18.9 \\
\hline A2063 & 249511419 & 18.0 \\
\hline A2063 & 250975083 & 16.9 \\
\hline A2063 & 333842971 & 12.8 \\
\hline A2063 & 348468092 & 14.1 \\
\hline A3581 & 242221372 & 16.8 \\
\hline A3581 & 252236390 & 11.7 \\
\hline A3581 & 272025793 & 15.8 \\
\hline A3581 & 292681335 & 12.2 \\
\hline A3581 & 300957764 & 11.9 \\
\hline
\end{tabular}

\section{References}

Abdo, A. A., Ackermann, M., Ajello, M., et al. 2009a, ApJ, 699, 31 Abdo, A. A., Ackermann, M., Ajello, M., et al. 2009b, ApJ, 707, 55 Abdo, A. A., Ackermann, M., Ajello, M., et al. 2010, Phys. Rev. Lett., 104, 101101

Ackermann, M., Ajello, M., Allafort, A., et al. 2010a, ApJ, 717, L71 Ackermann, M., Ajello, M., Allafort, A., et al. 2010b, JCAP, 5, 25
Ackermann, M., Ajello, M., Albert, A., et al. 2012a, ApJS, 203, 4 Ackermann, M., Ajello, M., Atwood, W. B., et al. 2012b, ApJ, 750, 3 Ackermann, M., Ajello, M., Allafort, A., et al. 2013, ApJS, 209, 34 Ackermann, M., Ajello, M., Albert, A., et al. 2014, ApJ, 787, 18 Ando, S., \& Nagai, D. 2012, JCAP, 7, 17

Araudo, A. T., Cora, S. A., \& Romero, G. E. 2008, MNRAS, 390, 323 Atwood, W. B. E. A. 2007, Astropart. Phys., 28, 422

Atwood, W. B., Abdo, A. A., Ackermann, M., et al. 2009, ApJ, 697, 1071

Bagchi, J., Durret, F., Neto, G. B. L., \& Paul, S. 2006, Science, 314, 791

Berezinsky, V. S., Blasi, P., \& Ptuskin, V. S. 1997, ApJ, 487, 529

Böhringer, H., Matsushita, K., Churazov, E., Ikebe, Y., \& Chen, Y. 2002, A\&A, 382,804

Brunetti, G., \& Lazarian, A. 2007, MNRAS, 378, 245

Chen, Y., Reiprich, T. H., Böhringer, H., Ikebe, Y., \& Zhang, Y.-Y. 2007, A\&A, 466, 805

Churazov, E., Forman, W., Jones, C., \& Böhringer, H. 2000, A\&A, 356, 788

Churazov, E., Forman, W., Vikhlinin, A., et al. 2008, MNRAS, 388, 1062

David, L. P., Slyz, A., Jones, C., et al. 1993, ApJ, 412, 479

David, L. P., Nulsen, P. E. J., McNamara, B. R., et al. 2001, ApJ, 557, 546

Dermer, C. D. 1986, A\&A, 157, 223

Drury, L. O., Aharonian, F. A., \& Voelk, H. J. 1994, A\&A, 287, 959

Dutson, K. L., White, R. J., Edge, A. C., Hinton, J. A., \& Hogan, M. T. 2013, MNRAS, 429, 2069

Ensslin, T. A., Biermann, P. L., Kronberg, P. P., \& Wu, X.-P. 1997, ApJ, 477, 560

Ferrari, C., Govoni, F., Schindler, S., Bykov, A. M., \& Rephaeli, Y. 2008, Space Sci. Rev., 134, 93

Finke, J. D., Razzaque, S., \& Dermer, C. D. 2010, ApJ, 712, 238

Franceschini, A., Rodighiero, G., \& Vaccari, M. 2008, A\&A, 487, 837

Healey, S. E., Romani, R. W., Cotter, G., et al. 2008, ApJS, 175, 97

Hoekstra, H., Donahue, M., Conselice, C. J., McNamara, B. R., \& Voit, G. M. 2011, ApJ, 726, 48

Huber, B., Tchernin, C., Eckert, D., et al. 2013, A\&A, 560, A64

Inoue, S., Aharonian, F. A., \& Sugiyama, N. 2005, ApJ, 628, L9

Kneiske, T. M., Mannheim, K., \& Hartmann, D. H. 2002, A\&A, 386, 1

Li, T.-P., \& Ma, Y.-Q. 1983, ApJ, 272, 317

Mattox, J. R., Bertsch, D. L., Chiang, J., et al. 1996, ApJ, 461, 396

Michelson, P. F., Atwood, W. B., \& Ritz, S. 2010, Rep. Prog. Phys., 73, 074901

Miniati, F. 2003, MNRAS, 342, 1009

Mittal, R., Hudson, D. S., Reiprich, T. H., \& Clarke, T. 2009, A\&A, 501, 835

Moiseev, A. A., Hartman, R. C., Ormes, J. F., et al. 2007, Astropart. Phys., 27, 339

Nolan, P. L., Abdo, A. A., Ackermann, M., et al. 2012, ApJS, 199, 31

Peterson, J. R., Paerels, F. B. S., Kaastra, J. S., et al. 2001, A\&A, 365, L104

Peterson, J. R., Kahn, S. M., Paerels, F. B. S., et al. 2003, ApJ, 590, 207

Petrosian, V., Bykov, A., \& Rephaeli, Y. 2008, Space Sci. Rev., 134, 191

Pinzke, A., \& Pfrommer, C. 2010, MNRAS, 409, 449

Reimer, O., Pohl, M., Sreekumar, P., \& Mattox, J. R. 2003, ApJ, 588, 155

Reiprich, T. H., \& Böhringer, H. 2002, ApJ, 567, 716

Sarazin, C. L. 1986, Rev. Mod. Phys., 58, 1

Skellam, J. G. 1952, Biometrica, 39, 346

Thompson, D. J. 2008, Rep. Prog. Phys., 71, 116901

Truemper, J. 1993, Science, 260, 1769

Voges, W., Aschenbach, B., Boller, T., et al. 1999, A\&A, 349, 389

Völk, H. J., Aharonian, F. A., \& Breitschwerdt, D. 1996, Space Sci. Rev., 75, 279

Wilks, S. 1962, Mathematical Statistics (New York: Wiley)

Zimmer, S., Conrad, J., for the Fermi-LAT Collaboration, \& Pinzke, A. 2011, Contribution to the 2011 Fermi Symposium - eConf Proceedings C110509 [arXiv: 1110.6863] 